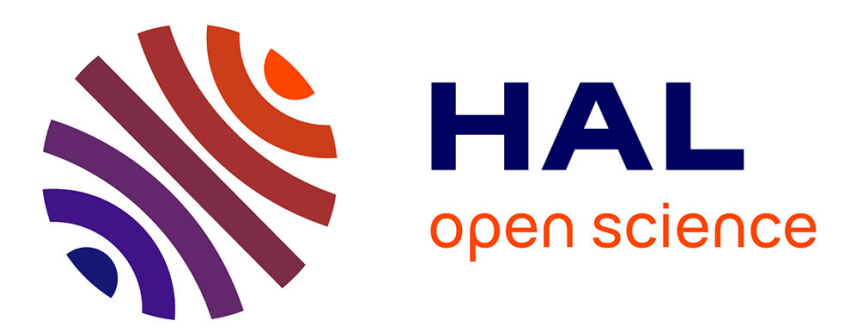

\title{
RBF approximation by partition of unity for valuation of options under exponential Lévy processes
}

\author{
Ali Fereshtian, Reza Mollapourasl, Florin Avram
}

\section{To cite this version:}

Ali Fereshtian, Reza Mollapourasl, Florin Avram. RBF approximation by partition of unity for valuation of options under exponential Lévy processes. Journal of computational science, 2019, 32, pp.44-55. 10.1016/j.jocs.2019.02.008 . hal-02369204

\section{HAL Id: hal-02369204 https://hal.science/hal-02369204}

Submitted on 18 Nov 2019

HAL is a multi-disciplinary open access archive for the deposit and dissemination of scientific research documents, whether they are published or not. The documents may come from teaching and research institutions in France or abroad, or from public or private research centers.
L'archive ouverte pluridisciplinaire HAL, est destinée au dépôt et à la diffusion de documents scientifiques de niveau recherche, publiés ou non, émanant des établissements d'enseignement et de recherche français ou étrangers, des laboratoires publics ou privés. 


\section{Manuscript Details}

\section{Manuscript number}

Title
JOCS_2018_1019

RBF approximation by partition of unity for valuation of options under exponential LI' $\{$ e\}vy processes

Full Length Article

\section{Article type}

Abstract

The prices of some European and American-style contracts on assets driven by a class of Markov processes containing, in particular, LI'\{e\}vy processes of pure jump type with infinite jump activity, are obtained numerically, as solutions of the partial integro-differential equations (PIDEs) they satisfy. This paper overcomes the ill-conditioning inherent in global meshfree methods by using localized RBF approximations known as the RBF partition of unity (RBFPU) method for (PIDEs) arising in option pricing problems in LI'\{e\}vy driven assets. Then, Crank-Nicolson, Leap-Frog (CNLF) is applied for time discretization. We treat the local term using an implicit step, and the nonlocal term using an explicit step, to avoid the inversion of the nonsparse matrix. For dealing with early exercise feature of American option and solving free boundary problem we use the implicit-explicit method combined with a penalty method. Efficiency and practical performance are demonstrated by numerical experiments for pricing European and American contracts.

Keywords

Corresponding Author

Order of Authors

Suggested reviewers
Radial basis functions, partition of unity, Option pricing, LI'\{e\}vy processes, Markov processes

Reza Mollapourasl

Ali Fereshtian, Reza Mollapourasl, Florin Avram

Mohan Kadalbajoo, Mehdi Dehghan, simon hubbert

\section{Submission Files Included in this PDF}

File Name [File Type]

cover letter.pdf [Cover Letter]

Research Highlights.pdf [Highlights]

Levy_Fereshtian.pdf [Manuscript File]

\section{Submission Files Not Included in this PDF}

File Name [File Type]

Paper_Levy.zip [LaTeX Source File]

To view all the submission files, including those not included in the PDF, click on the manuscript title on your EVISE Homepage, then click 'Download zip file'. 
Dear Editor,

We would like to submit our manuscript entitled

\section{RBF approximation by partition of unity for valuation of options under exponential Lévy processes}

for exclusive consideration of publication as a research paper in Journal of Computational Science.

The prices of some European and American-style contracts on assets driven by a class of Markov processes containing, in particular, Lévy processes of pure jump type with infinite jump activity, are obtained numerically, as solutions of the partial integro-differential equations (PIDEs) they satisfy. This paper overcomes the ill-conditioning inherent in global meshfree methods by using localized RBF approximations known as the RBF partition of unity (RBF-PU) method for (PIDEs) arising in option pricing problems in Lévy driven assets. Then, Crank-Nicolson, Leap-Frog (CNLF) is applied for time discretization. We treat the local term using an implicit step, and the nonlocal term using an explicit step, to avoid the inversion of the nonsparse matrix. For dealing with early exercise feature of American option and solving free boundary problem we use the implicit-explicit method combined with a penalty method. Efficiency and practical performance are demonstrated by numerical experiments for pricing European and American contracts.

Thank you for considering our paper. We appreciate your effort and look forward to your response.

With best regards

Ali Fereshtian

Reza Mollapourasl (corresponding author, mollapor@oregonstate.edu)

Florin Avram 


\section{Research Highlights}

We proposed RBF-PU method for spatial discretization of PIDE to price American and European options under Lévy model. Also, penalty method is used to solve the free boundary problem arisen in pricing American option. Then, CNLF is applied for time discretisations and we treat the local term using an implicit step and the nonlocal term using an explicit step to avoid to avoid the inversion of the nonsparse matrix. These result in a linear algebraic system with a sparse matrix that has a small condition number. The shape parameter in the RBF affects the accuracy and stability of the numerical methods. Numerical results confirm that RBF-PU method is less sensitive to the change of shape parameter. An increase of the number of nodal points and correspondingly an increase of the number of patches in RBF-PU method also leads to an improvement of the approximation. The effect of the time discretization is measured by studying the temporal error. For the American and European option cases where the CNLF scheme is combined with penalty method, we conclude that the rate of convergence is of at least first order in time for more cases. Also, numerical results confirm that our scheme is stable and second order convergent in space. The experiments also demonstrated that European and American option prices with error around $1.0 e-4$ can be computed in less than one second on a PC. Thus, the developed method is very fast and accurate. 


\title{
RBF approximation by partition of unity for valuation of options under exponential Lévy processes
}

\author{
Ali Fereshtian ${ }^{1}$, Reza Mollapourasl ${ }^{2}$, and Florin Avram ${ }^{3}$ \\ ${ }^{1}$ School of Mathematics, Shahid Rajaee Teacher Training University, Lavizan, Tehran 16788, Iran \\ ${ }^{2}$ Department of Mathematics, Oregon State University, Corvallis, OR 97331, USA \\ ${ }^{3}$ Laboratoire de Mathématiques Appliquées, Université de Pau, France \\ a.fereshtian@sru.ac.ir, mollapor@oregonstate.edu, florin.avram@univ-pau.fr
}

\begin{abstract}
The prices of some European and American-style contracts on assets driven by a class of Markov processes containing, in particular, Lévy processes of pure jump type with infinite jump activity, are obtained numerically, as solutions of the partial integro-differential equations (PIDEs) they satisfy. This paper overcomes the ill-conditioning inherent in global meshfree methods by using localized RBF approximations known as the RBF partition of unity (RBF-PU) method for (PIDEs) arising in option pricing problems in Lévy driven assets. Then, Crank-Nicolson, Leap-Frog (CNLF) is applied for time discretization. We treat the local term using an implicit step, and the nonlocal term using an explicit step, to avoid the inversion of the nonsparse matrix. For dealing with early exercise feature of American option and solving free boundary problem we use the implicit-explicit method combined with a penalty method. Efficiency and practical performance are demonstrated by numerical experiments for pricing European and American contracts.
\end{abstract}

Keywords:Radial basis functions, partition of unity, Option pricing, Lévy processes, Markov processes

\section{Introduction}

It is widely recognized that the classic option pricing model proposed in 1973 by Black and Scholes in [6] and Merton in [35], does not ideally fit observed empirical market data. Two identified empirical features have been under much attention, the first one is skewed distribution with higher peak and heavier tails of the return distribution and the second one is the volatility smile [2]. Jumps are regularly observed in the discrete movement of stock price and these jumps can not be captured by the log normal distribution characteristic of the stock price in the Black Scholes model. Therefore an alternative model is necessary to overcome these issued. To resolve these, issue several models have been proposed in the literature. Among these, the jump diffusion model introduced by Merton [35] and Kou [30] is one of the most used model. Merton proposed a log-normally distributed process for the jump-amplitudes, while Kou suggested logdouble-exponentially distributed process. 
These models have finite jump activity, unlike the more general approach with possibly infinite jump activity - see $[12,18]$. Another motivation for our study comes from stochastic volatility models with jumps. The model proposed by Bates in [5] with jumps only in the value of the underlying asset is an example of such an approach. More general jump-diffusion models with stochastic volatility are considered in [22], for example.

A variety of numerical techniques have been proposed for pricing options under both models with stochastic volatility and models with jumps. In particular, in the case of models with jumps, we mention the paper by Matache et al. [34], where a finite element method is developed, the papers by Andersen and Andreasen [2], by Cont and Voltchkova [19], by d'Halluin et al. [21, 52], and by Clift and Forsyth [17], where finite difference schemes are presented, and the papers by Fang and Oosterlee $[23,24]$, where a numerical method based on the characteristic function is proposed.

In models with jumps, the non local integral term leads to dense matrices after discretization [19] and efficient numerical methods are required for pricing of complex contracts and for calibration of model parameters. This fact means that fully implicit schemes encounter difficulties such as dense matrix inversions whereas fully explicit schemes impose stability restrictions [8]. For some solutions to this problem in $[20,32]$ authors have used an iterative procedure for solving the discretized equations, and more efficient approaches using implicit-explicit Runge-Kutta schemes which treat the integral term explicitly were later proposed by Cont and Voltchkova in [19] and by Briani et al. [8]. Also, an unconditionally stable alternating direction implicit (ADI) method was proposed by Andersen and Andreasen in [2].

Option pricing using RBFs was explored in one dimension for European and American options by Hon et al. [28], [51]. Pettersson et al. [39] presented an RBF based method for multi-dimensional European options, and American options in both one and two dimensions are investigated by Fasshauer et al. [26]. All of these papers employ global RBF collocation methods. Global RBF collocation methods lead to dense linear systems, and computational costs that become prohibitive as the number of dimensions increase. In order to address the computational cost issues of the global RBF method, we need to introduce locality. An easy way to do that is to use compactly supported RBFs, such as the Wendland functions [48], but then the spectral convergence properties are lost. Here we take another approach, where the infinitely smooth RBFs are still used in the approximation but over local subregions of the computational domain [37]. The possibility of using RBFs in a partition of unity scheme was mentioned in [4], further discussed in [49], implemented for interpolation on the sphere, in the plane, and in three-dimensional space in $[15,16]$. Also, we applied localized partition of unity method for pricing American and European option prices as well as Greeks in [36].

We introduce below a local method for option pricing problems under Lévy processes, based on radial basis function partition of unity (RBF-PU) approximation. Some advantages of partition of unity method based on radial basis function are the flexibility in choosing ansatz spaces and their good approximation properties. Also, this method helps us to have an sparse system with low condition number and control the computational cost and run time of the algorithm. The outline of the paper is as follows.

In the next section, we describe Lévy's model and partial integro-differential equations (PIDEs) for pricing European options. Also, American option is formulated as linear complimentary problem (LCP) then we apply penalty method to solve it. In section 3, the basics of radial basis functions as well as the partition of unity method based on RBFs and the definition of the differentiation matrices are introduced. In section 4, RBF-PU method is used to discetize differential operator 
and for integral operator, we use trapezoid rule. Then, (CNLF) is applied for time discretization and we treat the local term using an implicit step and the nonlocal term using an explicit step to avoid the inversion of the nonsparse matrix. Finally, in the last section, the accuracy and efficiency of the proposed method is numerically investigated for European and American options.

\section{Option Pricing under the Exponential Lévy Model}

Observation of sudden, large movements in the prices of financial assets has led to the use of stochastic processes with discontinuous trajectories - jump processes - as models for financial assets. Exponential Lévy models [18] offer analytically tractable examples of positive processes with jumps which are simple enough to allow a detailed study, and yet complex enough to allow calibrating the model to market prices of options. The Markov property of the price implies that option prices are solutions of partial integro-differential equations (PIDEs) which involve, in addition to a (possibly degenerate) second-order differential operator, a non-local integral term which requires specific treatment both at the theoretical and numerical level.

We consider here the class of models where the risk neutral dynamics of the underlying asset is given by $S_{t}=S_{0} \exp \left((r-q) t+X_{t}\right)$ where $X_{t}$ is a Lévy process with a Lévy triplet $\left(\sigma^{2}, \gamma, \nu\right)$. The law of Lévy process is completely determined by its characteristic triplet $\left(\sigma^{2}, \gamma, \nu\right)$, where $\sigma^{2}$ is called Gaussian variance, $\gamma$ is related to the drift, and the Lévy measure satisfies the following condition

$$
\int_{\mathbb{R}} \min \left(1, x^{2}\right) \nu(d x)<\infty .
$$

The probabilistic interpretation of $\nu$ is that $\nu(d x)$ gives the expected number of jumps with size between $x$ and $x+d x$, which the process makes between time 0 and 1 . An infinite activity Lévy process is described by an infinite number of jumps, that is, $\nu(\mathbb{R})=\infty$.

Example 1. The CGMY process [13] is an infinite activity processes defined by the Levy measure

$$
\nu(d x)= \begin{cases}\frac{C e^{-G|x|}}{|x|^{1+Y}} d x, & x<0 \\ \frac{C e^{-M x}}{x^{1+Y}} d x, & x>0\end{cases}
$$

for constants $C>0, G \geq 0, M \geq 0$, and $0<Y<2$.

Example 2. In the case $Y=0$, a special choice of the exponents $G, M$ yields the subclass of Variance-Gamma or VG processes, which were introduced earlier by Madan and Seneta (1990) [33] as time changed Brownian motions.

The standard tool to assign prices to options under Lévy processes consists in changing to a convenient probability measure and taking the expectation of the discounted prices and define the pricing function $v$ driven by $X_{t}$ by

$$
v(\tau, x)=e^{-r \tau} \mathbb{E}\left[g\left(x+(r-q) \tau+X_{\tau}\right)\right]
$$


where $g(\cdot)$ is the payoff function, $\tau=T-t$ is the time to maturity $T$, and $x=\log \left(\frac{S}{K}\right)$ is the $\log$ price of the underlying asset $S$.

Replace now the infinitely many jumps smaller than $\epsilon$ by a Brownian motion, leaving only the jumps whose magnitudes are larger than $\epsilon$, which are driven by a finite activity Levy model [3]. To be specific, we define its corresponding Levy process $\bar{X}_{t}$ that has the Levy triplet $\left(\bar{\sigma}^{2}, \bar{\gamma}, \bar{\nu}\right)$ given by

$$
\begin{gathered}
\bar{\sigma}^{2}=\sigma^{2}+\int_{-\epsilon}^{\epsilon} z^{2} \nu(d z) \\
\bar{\nu}=\nu \mathbf{1}_{|x| \geq \epsilon} \\
\bar{\gamma}=-\frac{1}{2} \sigma^{2}-\int_{\mathbb{R}}\left(e^{x}-1-x \mathbf{1}_{|x| \leq 1}\right) \bar{\nu}(d x)
\end{gathered}
$$

where $\mathbf{1}$ is the indicator function. Now let

$$
u(\tau, x)=e^{-r \tau} \mathbb{E}\left[g\left(x+(r-q) \tau+\bar{X}_{\tau}\right)\right]
$$

where $u(\tau, x)$ satisfies the following partial integro-differential equation(PIDE)

$$
\begin{cases}\partial_{\tau} u-\mathcal{P} u=0, & \tau \in(0, T] \\ u(0, x)=g(x), & x \in \mathbb{R}\end{cases}
$$

where the integro-differential operator $\mathcal{P}$ is

$\mathcal{P} u(\tau, x)=\frac{1}{2} \bar{\sigma}^{2} \partial_{x}^{2} u(\tau, x)+\left(r-q-\frac{\bar{\sigma}^{2}}{2}-\lambda(\epsilon) \xi(\epsilon)\right) \partial_{x} u(\tau, x)-(r+\lambda(\epsilon)) u(\tau, x)+\int_{|y| \geq \epsilon} u(\tau, x+y) \nu(d y)$ where $\lambda(\epsilon)=\int_{|z| \geq \epsilon} \nu(d z), \xi(\epsilon)=\frac{1}{\lambda(\epsilon)} \int_{|z| \geq \epsilon}\left(e^{z}-1\right) \nu(d z)$.

Theorem 1. Let $g$ be Lipschitz: $|g(x)-g(y)| \leq K|x-y|$. Let $v(\tau, x)$ and $u(\tau, x)$ be defined by $(2)$ and (4), respectively. Then

$$
|v(\tau, x)-u(\tau, x)| \leq C \frac{\int_{|y| \leq \epsilon}|y|^{3}(d y)}{\bar{\sigma}^{2}}
$$

Proof. [19]

Remark 1. If $\lim _{x \rightarrow 0} \nu(x)|x|^{1+\beta}=a>0$ with $0 \leq \beta<2$, then (5) gives

$$
|v(\tau, x)-u(\tau, x)| \leq C(\beta) \epsilon
$$

so the approximation error is proportional to $\epsilon$. This case includes all practical examples used in option pricing such as variance Gamma, NIG, and tempered stable processes [19].

Example 3. Two important examples of payoff functions are

$$
g(x)= \begin{cases}\max \left(K-K e^{x}, 0\right), & \text { for put option } \\ \max \left(K e^{x}-K, 0\right), & \text { for call option } .\end{cases}
$$

In the remaining of this paper we will focus on put options, and as additional boundary conditions we impose

$$
\lim _{x \rightarrow-\infty} u(\tau, x)=K e^{-r \tau}, \quad \lim _{x \rightarrow \infty} u(\tau, x)=0 .
$$


For American options, it is well known that for each time $\tau \in[0, T]$, there always exists a stock price $x$ for which early exertion before final time $T$ is advantageous [43]. Therefore the American option pricing problem can be formulated in the form of an inequality by adopting the linear complementarity problem (LCP) formulation

$$
\left\{\begin{array}{l}
\partial_{\tau} u(\tau, x)-\mathcal{P} u(\tau, x) \geq 0, \\
u(0, x) \leq g(x), \\
\left(\partial_{\tau} u(\tau, x)-\mathcal{P} u(\tau, x)\right)(u(0, x)-g(x))=0,
\end{array}\right.
$$

for $(\tau, x) \in \mathbb{R} \times(0, T]$ and we need to impose the following boundary conditions

$$
\lim _{x \rightarrow-\infty} u(\tau, x)=K, \quad \lim _{x \rightarrow \infty} u(\tau, x)=0 .
$$

For solving the free boundary problem, there are some techniques such as linear programming [7] or penalty methods presented in [52, 41, 44]. Also, an other method for pricing American options is the operator splitting method introduced by Ikonen and Toivanen in [29] for American put option under the Black-Scholes model, and the method is studied by Toivanen in [46] under the Kou model. In this study, we consider penalty method for solving (LCP) problem (8), so we use penalty term defined by

$$
\frac{\delta C}{u(\tau, x)+\delta-q(x)}
$$

where $0<\delta \ll 1$ is a small regularization parameter, $C \geq r K$ is a positive constant and $q(x)=$ $K-K e^{x}$ then we replace problem (8) by the nonlinear PIDE

$$
\left\{\begin{array}{l}
\partial_{\tau} u(\tau, x)-\mathcal{P} u(\tau, x)-\frac{\delta C}{u(\tau, x)+\delta-q(x)}=0, \\
u(0, x)=g(x),
\end{array}\right.
$$

for $(\tau, x) \in \mathbb{R} \times(0, T]$ and also we need to impose boundary conditions (9).

\section{Approximation based on RBF-PUM}

To avoid mesh generation, in recent years meshless techniques have attracted the attention of researchers. In a meshless method a set of scattered nodes is used instead of meshing the domain of the problem. The technique of RBFs is one of the most recently developed meshless methods that has attracted attention of many researchers in recent years. Radial basis functions are an efficient tool for solving multivariate scattered data interpolation problems. To interpolate an unknown function $f \in C(\Omega)$ whose values on a set $X=\left\{\mathbf{x}_{1}, \mathbf{x}_{2}, \ldots, \mathbf{x}_{N}\right\} \subset \Omega \subset \mathbb{R}^{d}$ are known, a function of the form

$$
s_{f, X}(\mathbf{x})=\sum_{j=1}^{N} \lambda_{j} \phi\left(\left\|\mathbf{x}-\mathbf{x}_{j}\right\|\right),
$$

is chosen, where $\phi: \Omega \times \Omega \rightarrow \mathbb{R}$ is a radial basis function and $\|\cdot\|$ is the Euclidean norm. In Table 1 some globally supported RBFs are listed which are commonly employed in the literature. The positive constant $c$ appearing in RBFs is called the shape parameter which dictates the flatness of the radial basis function and also has a key role on the convergence rate of the approximations and the condition number of the coefficient matrices. For more details about basic properties and 
types of radial basis functions, compactly and globally supported and also their wide applications in scattered date interpolations, the interested reader would be referred to recent works in this topic $[25,40,9,10]$.

\begin{tabular}{ll}
\hline Name of function & Definition \\
\hline Thin plate splines (TPS) & $(-1)^{k+1} r^{2 k} \log (r)$ \\
Gaussian (GA) & $\exp \left(-c^{2} r^{2}\right)$ \\
Invers multiquadrics (IMQ) & $\frac{1}{\sqrt{(c r)^{2}+1}}$ \\
multiquadrics (MQ) & $\sqrt{(c r)^{2}+1}$ \\
Conical splines & $r^{2 k+1}$ \\
\hline
\end{tabular}

Table 1: Some well-known functions that generate globally supported RBFs

RBFs methods have many advantages but generally coefficients matrix are dense, hence complexity of computations is expensive. In this section we introduces partition of unity method (PUM) which puts a given set of local approximation spaces together to produce a conforming global approximation.

Let $\Omega \subset \mathbb{R}^{d}$ be a bounded set, and let a covering $\left\{\Omega_{j}\right\}_{j=1}^{M}$ of the region $\Omega$ such that $\Omega \subset \bigcup_{j=1}^{M} \Omega_{j}$. Also, we define

$$
\forall \mathbf{x} \in \Omega \quad I(\mathbf{x}):=\left\{j \mid \mathbf{x} \in \Omega_{j}\right\}, \operatorname{card}(I(\mathbf{x})) \leq K,
$$

where the constant $K$ is independent of the number of patches $M$.

Definition 1. Let $\Omega \subset \mathbb{R}^{d}$ be a bounded set. Let $\left\{\Omega_{j}\right\}_{j=1}^{M}$ be an open and bounded covering of $\Omega$. This means all $\Omega_{j}$ are open and bounded and $\Omega$ is contained in their union. Set $\delta_{j}=\operatorname{diam}\left(\Omega_{j}\right)=$ $\sup _{x, \boldsymbol{y} \in \Omega_{j}}\|\boldsymbol{x}-\boldsymbol{y}\|_{2}$. A family of non-negative functions $\left\{w_{j}(\boldsymbol{x})\right\}_{j=1}^{M}$ with $w_{j}(\boldsymbol{x}) \in C^{k}\left(\mathbb{R}^{d}\right)$ is a k-stable partition of unity respect to cover of $\Omega_{j}$ if:

1. $\operatorname{supp}\left(w_{j}\right) \subseteq \bar{\Omega}_{j}$

2. $\sum_{j} w_{j}(\boldsymbol{x})=1$ for $\boldsymbol{x} \in \Omega$

3. For every $\alpha \in \mathbb{N}_{0}^{d}$ with $|\alpha| \leq k$ there exist a constant $C_{\alpha}>0$ such that for all $1 \leq j \leq M$

$$
\left\|D^{\alpha} w_{j}\right\|_{L_{\infty}\left(\Omega_{j}\right)} \leq \frac{C_{\alpha}}{\delta_{j}^{\alpha}}
$$

The weight functions $w_{j}$ are constructed by using Shepard's method [45] as follow:

$$
w_{j}(\mathbf{x})=\frac{\phi_{j}(\mathbf{x})}{\sum_{k \in I(\mathbf{x})} \phi_{k}(\mathbf{x})}, \quad j=1,2, \ldots, M
$$

where $\phi_{j}(\mathbf{x})$ are compactly supported functions with support on $\Omega_{j}$. To guarantee non-negativity and compact support in $\Omega_{j}$, we define in (12)

$$
\phi_{j}(\mathbf{x})=\phi\left(\frac{\left\|\mathbf{x}-\mathbf{c}_{j}\right\|}{r_{j}}\right), \quad j=1,2, \ldots, M,
$$


where $\left\{\mathbf{c}_{j}\right\}_{j=1}^{M}$ and $\left\{r_{j}\right\}_{j=1}^{M}$ are the centers and radiuses of the circular, spherical or hyper-spherical patches $\left\{\Omega_{j}\right\}_{j=1}^{M}$ and where $\phi$ is one of the compactly supported functions with minimal degree described in [48, Corololary 9.14]. Here we consider the Wendland compact supported function [50]

$$
\phi(r)=(1-r)_{+}^{4}(4 r+1),
$$

which belongs to $C^{2}$ for the construction of the weight functions.

The global approximation function $s_{u}(\mathbf{x})$, with $\mathbf{x} \in \Omega$, to the function $u(\mathbf{x})$ is constructed as

$$
s_{u}(\mathbf{x})=\sum_{j=1}^{M} w_{j}(\mathbf{x}) s_{u, j}(\mathbf{x})=\sum_{j \in I(\mathbf{x})} w_{j}(\mathbf{x}) s_{u, j}(\mathbf{x}),
$$

where $s_{u, j}$ are local interpolants such that $s_{u, j}\left(\mathbf{x}_{i}\right)=u\left(\mathbf{x}_{i}\right)$ for each node $\mathbf{x}_{i} \in \Omega_{j}$. Then, the global PU approximant inherits the interpolation property, i.e. $s_{u}\left(\mathbf{x}_{i}\right)=u\left(\mathbf{x}_{i}\right)$. Using the cardinal basis functions

$$
\psi_{j}\left(\mathbf{x}_{i}\right)=\left\{\begin{array}{ll}
1, & i=j \\
0, & i \neq j
\end{array} \quad i=1,2, \ldots, N\right.
$$

the local interpolant $s_{u, j}(\mathbf{x})$ is an RBF-approximant of type $s_{u}(\mathbf{x})=\Psi(\mathbf{x}) \mathbf{u}$, on $\Omega_{j}$ where $\Psi(\mathbf{x})=\left[\psi_{1}(\mathbf{x}), \psi_{2}(\mathbf{x}), \cdots, \psi_{N}(\mathbf{x})\right]$.

For time dependent function $u(t, \mathbf{x})$, we construct the global approximant built up from local $\mathrm{RBF}$ interpolants of type

$$
s_{u}(t, \mathbf{x})=\sum_{j=1}^{N} \psi_{j}(\mathbf{x}) u_{j}(t)=\Phi(\mathbf{x}) \mathbf{A}^{-1} \mathbf{u}(t) .
$$

For $j \in\{1, \ldots, M\}$, let $J\left(\Omega_{j}\right):=\left\{k \mid \mathbf{x}_{k} \in \Omega_{j}\right\}$ be the set of indices of the node points that belong to the patch $\Omega_{j}$. For such patch $\Omega_{j}$, the local RBF approximation is given by

$$
s_{u, j}(t, \mathbf{x})=\sum_{k \in J\left(\Omega_{j}\right)} \psi_{k}(\mathbf{x}) u_{k}(t)
$$

where $s_{u, j}\left(t, \mathbf{x}_{k}\right)=u_{k}(t)$ for all nodes $\mathbf{x}_{k} \in \Omega_{j}$ and $\psi_{k}$ are cardinal basis functions. Hence, in the RBF-PUM, we obtain the global approximant for the time-dependent function $u(t, \mathbf{x})$

$$
s_{u}(t, \mathbf{x})=\sum_{j \in I(\mathbf{x})} w_{j}(\mathbf{x}) s_{u, j}(t, \mathbf{x})=\sum_{j \in I(\mathbf{x})} \sum_{k \in J\left(\Omega_{j}\right)} w_{j}(\mathbf{x}) \psi_{k}(\mathbf{x}) u_{k}(t) .
$$

Now, for deriving an error estimation for partition of unity method, we need the following definitions

Definition 2. For a bounded region $\Omega$ fill distance on $X=\left\{\boldsymbol{x}_{1}, \boldsymbol{x}_{2}, \ldots, \boldsymbol{x}_{N}\right\}$ define as following

$$
h_{X, \Omega}=\sup _{x \in \Omega} \min _{\boldsymbol{x}_{j} \in X}\left\|\boldsymbol{x}-\boldsymbol{x}_{j}\right\|_{2},
$$

which can be explained as largest distance that for every $\boldsymbol{x} \in \Omega$ there is one point $\boldsymbol{x}_{j}$ with this distance. 
Definition 3. A subdomain $\Omega_{j} \subseteq \mathbb{R}^{d}$ satisfies an interior cone condition if there exists an angle $\theta \in\left(0, \frac{\pi}{2}\right)$ and a radius $\gamma>0$ such that, for all $\boldsymbol{x} \in \Omega_{j}$, a unit vector $\xi(\boldsymbol{x})$ exists such that the cone

$$
\boldsymbol{C}(\boldsymbol{x}, \xi(\boldsymbol{x}), \theta, \gamma)=\left\{\boldsymbol{x}+\lambda \boldsymbol{y}: \boldsymbol{y} \in \mathbb{R}^{d},\|\boldsymbol{y}\|_{2}=1, \boldsymbol{y}^{T} \xi(\boldsymbol{x}) \geq \cos (\theta), \lambda \in[0, \gamma]\right\}
$$

is contained in $\Omega_{j}$.

Definition 4. Suppose $\Omega \subseteq \mathbb{R}^{d}$ is bounded and $X=\left\{\boldsymbol{x}_{1}, \boldsymbol{x}_{2}, \ldots, \boldsymbol{x}_{N}\right\} \subseteq \mathbb{R}^{d}$ are given. An open and bounded covering $\left\{\Omega_{j}\right\}_{j=1}^{M}$ is called regular for $(X, \Omega)$ if the following properties are satisfied

1. For each $x \in \Omega$, the number of subdomains $\Omega_{j}$, with $x \in \Omega_{j}$ is bounded by a global constant $C$,

2. Each subdomain $\Omega_{j}$ satisfies an interior cone condition,

3. The local fill distances $h_{X_{N_{j}}, \Omega_{j}}$ are uniformly bounded by the global fill distance $h_{X, \Omega}$.

For each positive definite function $\phi \in C^{k}\left(\mathbb{R}^{d}\right)$ and each area $\Omega \subset \mathbb{R}^{d}$ there is a function space $\mathcal{N}_{\phi}(\Omega)$, the native Hilbert space [42]. The smoothness of $\phi$ inherited from the native space via $\mathcal{N}_{\phi}(\Omega) \subseteq C^{\left[\frac{k}{2}\right]}(\Omega)$. Also, for getting the full approximation order, the weak form of Holder continuity idea is used, and we define space $C_{v}^{k}\left(\mathbb{R}^{d}\right)$ as space of all functions such that their derivatives of order $k$ satisfy $D^{\alpha} u(x)=\mathcal{O}\left(\|x\|_{2}^{v}\right)$ for $\|x\|_{2} \rightarrow 0$. By using the above definitions the following convergence theorem is derived from [50].

Theorem 2. Suppose $\phi \in C_{v}^{k}\left(\mathbb{R}^{d}\right)$ is conditionally positive definite of order $m$, also, let $\Omega \subseteq \mathbb{R}^{d}$ be open and bounded and $X=\left\{\boldsymbol{x}_{1}, \boldsymbol{x}_{2}, \ldots, \boldsymbol{x}_{N}\right\} \subseteq \Omega$. Let $\left\{\Omega_{j}\right\}_{j=1}^{M}$ be a regular covering for $(\Omega, X)$ and let $\left\{w_{j}\right\}_{j=1}^{M}$ be k-stable for $\left\{\Omega_{j}\right\}_{j=1}^{M}$. Then the error between $u \in \mathcal{N}_{\phi}(\Omega)$ and its partition of unity interpolant is bounded by

$$
\left|D^{\alpha} u(\boldsymbol{x})-D^{\alpha} s_{u}(\boldsymbol{x})\right| \leq C_{1} h_{X, \Omega}^{\frac{k+v}{2}-|\alpha|}|u|_{\mathcal{N}_{\phi}(\Omega)}
$$

for all $\boldsymbol{x} \in \Omega$ and all $|\alpha| \leq \frac{k}{2}$.

\section{Discretization}

In this section, we split the PIDE operator $\mathcal{P}$ into two parts as follows

$$
\mathcal{P} u(x, \tau)=\mathcal{D} u(x, \tau)+\mathcal{J} u(x, \tau),
$$

where $\mathcal{D}$ is a differential operator and $\mathcal{J}$ is an integral operator defined by

$$
\begin{gathered}
\mathcal{D} u(\tau, x)=\frac{1}{2} \bar{\sigma}^{2} \partial_{x}^{2} u(\tau, x)+\left(r-q-\frac{\bar{\sigma}^{2}}{2}-\lambda(\epsilon) \xi(\epsilon)\right) \partial_{x} u(\tau, x)-(r+\lambda(\epsilon)) u(\tau, x) \\
\mathcal{J} u(\tau, x)=\int_{|y| \geq \epsilon} u(\tau, x+y) \nu(y)(d y) .
\end{gathered}
$$

For the spatial variable $x$, the domain is restricted to a bounded set $\Omega=\left[x_{\min }, x_{\max }\right]$ and generate uniform grid points on the truncated region. For given positive integers $N$ and $M$, let 
$\triangle \tau=\frac{T}{M}$ and $\triangle x=\frac{x_{\text {max }}-x_{\text {min }}}{N-1}$. Now, let $\tau_{m}=m \triangle \tau$ for $m=0,1, \ldots, M$ and $x_{n}=x_{\text {min }}+(n-1) \triangle x$ for $n=1, \ldots, N$. We define $u\left(x_{n}, \tau_{m}\right)$ to be the price at the $n$th spatial and $m$ th temporal node.

To use the RBF-PUM approximation for spatial discretization of PIDE problems (4) and (10), we need to compute the effect of applying a spatial differential operator $\mathcal{D}$ at the interior node points. Let $L$ be a derivative operator of order $\alpha$. By applying of operator $L$ in RBF-PUM approximation for interior point we get

$$
\begin{aligned}
L u(\tau, x)=\frac{\partial^{|\alpha|}}{\partial x^{\alpha}} u(\tau, x) & =\sum_{i \in I(x)} \sum_{k \in J\left(\Omega_{i}\right)} \frac{\partial^{|\alpha|}}{\partial x^{\alpha}}\left[w_{i}(x) \psi_{k}(x)\right] u_{k}(\tau) \\
& =\sum_{i \in I(x)} \sum_{k \in J\left(\Omega_{i}\right)}\left[\sum_{\beta \leq \alpha}\left(\begin{array}{c}
\alpha \\
\beta
\end{array}\right) \frac{\partial^{|\alpha-\beta|}}{\partial x^{\alpha-\beta}} w_{i}(x) \frac{\partial^{|\beta|}}{\partial x^{\beta}} \psi_{k}(x)\right] u_{k}(\tau) .
\end{aligned}
$$

For composite linear operators like $\mathcal{D}$, we sum up the contributions from each term, and denote the global differentiation matrix under operator $\mathcal{D}$ by $\mathbf{D}$.

To numerical approximation of the integral operator $\mathcal{J}$ at each grid point $\left(\tau_{k}, x_{i}\right)$, we first use transformation $z=x+y$, then we have

$$
\mathcal{J} u\left(\tau_{k}, x_{i}\right)=\int_{\left|z-x_{i}\right| \geq \epsilon} u\left(\tau_{k}, z\right) \nu\left(z-x_{i}\right) d z
$$

Now, let $N$ as a constant to cut off the domain $\mathbb{R}$ to a bounded interval $\left(x_{-N}, x_{N}\right)$ where the values $x_{-N}$ and $x_{N}$ will be chosen based on standard financial arguments, and zero belongs to $\left[x_{-N}, x_{N}\right]$, and split the integral operator to the following four integrals

$$
\begin{aligned}
\mathcal{J} u\left(\tau_{k}, x_{i}\right) & =\int_{x_{-N}}^{x_{l_{i}}} u\left(\tau_{k}, z\right) \nu\left(z-x_{i}\right) d z+\int_{x_{l_{i}}}^{x_{i}-\epsilon} u\left(\tau_{k}, z\right) \nu\left(z-x_{i}\right) d z \\
& +\int_{x_{i}+\epsilon}^{x_{L_{i}+1}} u\left(\tau_{k}, z\right) \nu\left(z-x_{i}\right) d z+\int_{x_{L_{i}+1}}^{x_{N}} u\left(\tau_{k}, z\right) f\left(z-x_{i}\right) d z
\end{aligned}
$$

where $l_{i}$ and $L_{i}$ are the greatest integers such that

$$
x_{l_{i}}<x_{i}-\epsilon<x_{l_{i}+1}, \quad x_{L_{i}}<x_{i}+\epsilon<x_{L_{i}+1} .
$$

Now these integrals can be approximated using the trapezoidal rule and for evaluating the second and third integrals, we need the values of $u\left(\tau_{k}, x_{i}-\epsilon\right)$ and $u\left(\tau_{k}, x_{i}+\epsilon\right)$ to apply the trapezoidal rule. For doing this since $x_{l_{i}}<x_{i}-\epsilon<x_{l_{i}+1}$ and $x_{L_{i}}<x_{i}+\epsilon<x_{L_{i}+1}$, the values $u\left(\tau_{k}, x_{i}-\epsilon\right)$ and $u\left(\tau_{k}, x_{i}+\epsilon\right)$ can be approximated by using the linear interpolation on the intervals $\left[x_{l_{i}}, x_{l_{i}+1}\right]$ and $\left[x_{L_{i}}, x_{L_{i}+1}\right]$ respectively. Finally, by using approximate values for integrals, the integral matrix under integral operator $\mathcal{J}$ is derived and denoted by $\mathbf{J}$.

To evaluate an European option, let $\mathbf{U}_{m}:=\left[u\left(\tau_{m}, x_{1}\right), u\left(\tau_{m}, x_{2}\right), \cdots, u\left(\tau_{m}, x_{N}\right)\right]^{\top}$ (here $\top$ means transpose) as an approximate value of the solution which can be obtained by using the following Crank-Nicolson, Leap-Frog (CNLF) time stepping scheme

$$
\frac{\mathbf{U}_{m+1}-\mathbf{U}_{m-1}}{2 \triangle \tau}=\mathbf{D}\left(\frac{\mathbf{U}_{m+1}+\mathbf{U}_{m-1}}{2}\right)+\mathbf{J} \mathbf{U}_{m}, \quad 1 \leq m \leq M-1
$$


where $\mathbf{D}$ is the differentiation matrix associated with the differential operator and $\mathbf{J}$ is integral matrix corresponding to the integral operator. For $m=0$ we apply the following scheme

$$
\frac{\mathbf{U}_{m+1}-\mathbf{U}_{m}}{\triangle \tau}=\mathbf{D}\left(\frac{\mathbf{U}_{m+1}+\mathbf{U}_{m}}{2}\right)+\mathbf{J} \mathbf{U}_{m}
$$

Also, the initial vector is defined by $\mathbf{U}_{0}=\left[g\left(x_{1}\right), g\left(x_{2}\right), \cdots, g\left(x_{N}\right)\right]^{\top}$ where $g(\cdot)$ is the payoff function (6). One of the sources of error can arise when we use initial function (6) at grid points, since this function is discontinuous in their first derivatives. A useful notion in the implementation of numerical method is that the value of a function on a grid represents average value of the function over the surrounding grids rather than its value sampled at each grid point by

$$
g\left(x_{i}\right) \approx \frac{1}{\triangle x} \int_{x_{i}-\frac{\Delta x}{2}}^{x_{i}+\frac{\Delta x}{2}} g(x) d x,
$$

and this makes the payoff function smooth at the strike price $K$, and we use this technique to improve the accuracy of RBF-PU numerical method especially near the strike price.

For American option, after spatial discretization of differential and integral operators of (10), the solution can be obtained by using the following (CNLF) time stepping scheme

$$
\frac{\mathbf{U}_{m+1}-\mathbf{U}_{m-1}}{2 \triangle \tau}=\mathbf{D}\left(\frac{\mathbf{U}_{m+1}+\mathbf{U}_{m-1}}{2}\right)+\mathbf{J} \mathbf{U}_{m}+\mathbf{F}_{m}, \quad 0 \leq m \leq M-1
$$

where the initial vector is $\mathbf{U}_{0}=\left[g\left(x_{1}\right), g\left(x_{2}\right), \cdots, g\left(x_{N}\right)\right]^{\top}$ where $g(\cdot)$ is the payoff function (6), and

$$
\mathbf{F}_{m}=\left[\frac{\delta C}{u\left(\tau_{m}, x_{1}\right)+\delta-q\left(x_{1}\right)}, \frac{\delta C}{u\left(\tau_{m}, x_{2}\right)+\delta-q\left(x_{2}\right)}, \cdots, \frac{\delta C}{u\left(\tau_{m}, x_{N}\right)+\delta-q\left(x_{N}\right)}\right]^{\top} .
$$

\section{$5 \quad$ Numerical Results}

In this section, we present numerical results to show efficiency and performance of RBF-PU method and discuss more about convergence of proposed technique. All computations are carried in MAT$\mathrm{LAB}$ with a $3.6 \mathrm{GHz}$ Corei3 processor. Although the proposed scheme works for all radial basis functions from Table 1 we choose the multiquadric radial basis function for all the numerical experiments. We present absolute errors to check the efficiency and accuracy of RBF-PU method and for evaluating absolute error we have used reference solutions used in literature. Also, to show the practical performance of proposed numerical method, we report condition number of linear systems and CPU time in second.

The accuracy of RBF-methods where the differentiation matrix contains the differentiations of the shape function highly depends upon the shape parameter $c$ of the basis functions, which is responsible for the flatness of the functions. For smooth problems, the best accuracy is typically achieved when $c$ is small, but then the condition number of the linear system becomes very large. Figure 1 displays the dependence of root mean square (RMS) error on the size of the shape parameter for European and American options for some common test examples. For computing RMS error, we selected $N=2048$ and $M=2048$ as numbers of space and time steps and evaluated option price as a reference value for both European and American options in the region of interest $\left[\frac{K}{2}, \frac{3 K}{2}\right]$. We only considered parameter sets 2, 6 and 7 and other sets make similar results. Figure 1 shows that RBF-PU method is less sensitive to the change of shape parameter and we can derive that $c=2$ is a good choice for European options and $c=3$ is a good choice for American options. 


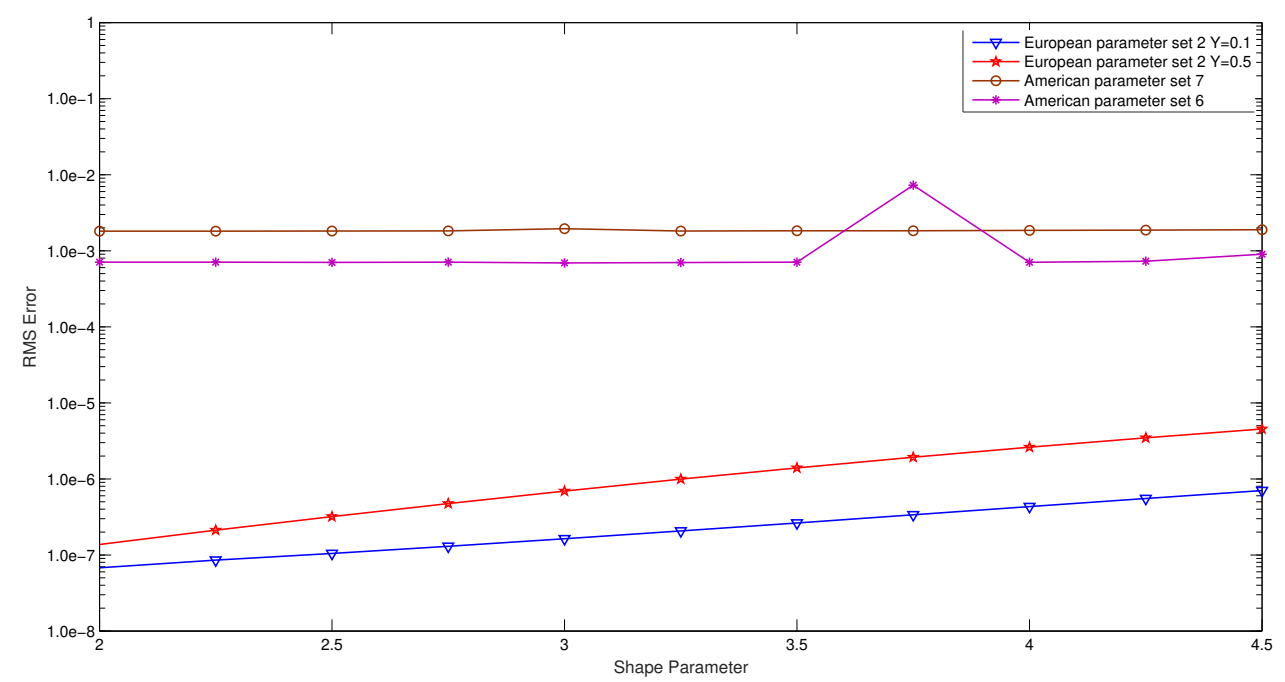

Figure 1: Root mean square (RMS) error as function of the shape parameter $c$ for European and American options.

\subsection{European options}

This section of numerical results consists in computing European put options with different sets of parameter values used in literature $[31,47,1]$ given in Table 2 in order to verify the convergence and stability of RBF-PU method combined with three time level (CNLF) discretization. We compare the numerical values with the Carr-Madan formula [14] as the reference option price.

We employed parameter set 1 to evaluate European put option and numerical results have been reported in Table 3. In [31] an implicit method is used to evaluate European and American options under Lévy model. The proposed methods solve partial integro-differential equations for European options and linear complementarity problems for American options via an operator splitting method involving linear systems with tridiagonal matrices. Also, in [31] parameter set 1 has been used for European put option and results reported in Table 3. Comparing results in Table 3 with Table 3 of [31] confirm that RBF-PU method is more accurate than proposed method in [31] and needs less time and space steps to reach an specific error level. Also, CPU times and condition numbers presented in Table 3 show the efficiency of RBF-PU method and overcome ill conditioning issue of global RBF methods. Parameter set 1 has been used in [47] and the resulting (PIDE) is then solved using a preconditioned BiCGSTAB method coupled with a fast Fourier transform. Results reported in Table 3 compare favorably with those in Table 8 of [47].

\begin{tabular}{llllllllll}
\hline & $r$ & $\sigma$ & $q$ & $C$ & $G$ & $M$ & $Y$ & $T$ & $K$ \\
\hline CGMY, parameter set 1, see [31,47] & 0.1 & 0 & 0 & 1 & 8.8 & 9.2 & 1.8 & 0.25 & 10 \\
CGMY, parameter set 2, see [1] & 0.1 & 0 & 0 & 1 & 5 & 5 & 0.1 & 1 & 1 \\
\hline
\end{tabular}

Table 2: Parameter setup for European put options.

Also we used parameter set 2 for different values of $Y$ to evaluate European put option and 


\begin{tabular}{|c|c|c|c|c|c|c|c|c|c|}
\hline & & \multicolumn{2}{|c|}{$S=9$} & \multicolumn{2}{|c|}{$S=10$} & \multicolumn{2}{|c|}{$S=11$} & \multirow[b]{2}{*}{ time(s) } & \multirow[b]{2}{*}{ cond } \\
\hline$N$ & $M$ & Price & Error & Price & Error & Price & Error & & \\
\hline 128 & 64 & 4.967873 & $2.9 \mathrm{e}-1$ & 4.673085 & $2.8 \mathrm{e}-1$ & 4.404182 & $2.6 \mathrm{e}-1$ & 0.006 & 53.20 \\
\hline 256 & 128 & 4.721715 & $5.2 \mathrm{e}-2$ & 4.438339 & $4.8 \mathrm{e}-2$ & 4.182342 & $4.4 \mathrm{e}-2$ & 0.04 & 131.56 \\
\hline 512 & 256 & 4.677214 & $8.3 \mathrm{e}-3$ & 4.396917 & 7.0e-3 & 4.143768 & $5.9 \mathrm{e}-3$ & 0.26 & 286.24 \\
\hline 1024 & 512 & 4.669093 & $2.4 \mathrm{e}-4$ & 4.389674 & $1.6 \mathrm{e}-4$ & 4.137306 & $5.2 \mathrm{e}-4$ & 1.68 & 617.86 \\
\hline \multicolumn{2}{|c|}{ Ref. Price [14] } & 4.668846 & & 4.389843 & & 4.137836 & & & \\
\hline \multicolumn{2}{|c|}{ Price in [31] } & 4.671721 & & 4.392112 & & 4.139657 & & & \\
\hline \multicolumn{2}{|c|}{ Price in [47] } & & & 4.3716708 & & & & & \\
\hline
\end{tabular}

Table 3: Price of European put options with parameter set 1 and truncated domain is $[-5,5]$ and $\epsilon=0.07$.

numerical results have been reported in Table 4. Parameter set 2 has been used in [1] and in this paper partial integro differential equation is discretized in space by the collocation method and in time by an explicit backward differentiation formula for evaluation of European option. Numerical results reported in Table 2 of [1], and by comparison accuracy and efficiency of RBF-PU method are confirmed.

\subsection{American options}

In this section, we consider parameter sets given in Table 5 for evaluation of American put option. For all test cases, the reference prices for the American options are calculated using the CONV method described in [32] and given in [38]. For American put option, we consider three different sets 3,4 and 5 which in these cases we let $Y \in(1,2), Y=0$ and $Y \in(0,1)$ respectively, and numerical results are given in Table 6 including option price and absolute error at $S=10$, CPU times in second and condition number of linear system of equations.

Also, to increase the rate of convergence in time and space for American option problems, we implement a Richardson extrapolation method defined by

$$
\frac{4 u\left(\frac{\triangle \tau}{2}, \frac{\triangle x}{2}\right)-u(\triangle \tau, \triangle x)}{3},
$$

where $u\left(\frac{\Delta \tau}{2}, \frac{\Delta x}{2}\right)$ refers to the approximate solution by using the RBF-PU method with time step size $\frac{\Delta \tau}{2}$ and spatial step size $\frac{\Delta x}{2}$ and $u(\triangle \tau, \triangle x)$ refers to the approximate solution with time step size $\triangle \tau$ and spatial step size $\triangle x$.

In [38] exponential time integration is used for solving American option pricing problem under infinite activity Lévy models with parameter sets 3,4 and 5 and the performance of this scheme is compared with the Crank-Nicolson method and an implicit-explicit method in conjunction with an extrapolation. In [38] the exponential time integration scheme is superior over the entire range of errors and reaches an accuracy of the order of $1.2 e-4$ in 22.197 seconds, whereas the CrankNicolson algorithm takes 58.669 seconds and the implicit-explicit extrapolation scheme reaches the same accuracy in 94.297 seconds. By comparing CPU times reported in Table 6, it is clear that RBF-PU method due to the sparsity of linear system is more faster than the proposed methods in $[38]$. 


\begin{tabular}{cccccc}
\hline \multicolumn{6}{c}{$S=1, Y=0.1$} \\
\hline$N$ & $M$ & Price & Error & time(s) & cond \\
\hline 128 & 64 & 0.06541121 & $1.8 \mathrm{e}-3$ & 0.003 & 2.74 \\
256 & 128 & 0.06393296 & $3.9 \mathrm{e}-4$ & 0.03 & 4.02 \\
512 & 256 & 0.06361654 & $8.2 \mathrm{e}-5$ & 0.27 & 6.52 \\
1024 & 512 & 0.06353348 & $5.5 \mathrm{e}-7$ & 1.82 & 12.57 \\
\hline Ref. Price $[14]$ & 0.06353404 \\
\hline \hline \multicolumn{7}{c}{$S=1, Y=0.5$} \\
\hline$N$ & $M$ & Price & Error & time(s) & cond \\
\hline 128 & 64 & 0.10627396 & $3.3 \mathrm{e}-3$ & 0.003 & 11.38 \\
256 & 128 & 0.10394723 & $9.8 \mathrm{e}-4$ & 0.04 & 24.05 \\
512 & 256 & 0.10315908 & $1.9 \mathrm{e}-4$ & 0.25 & 40.28 \\
1024 & 512 & 0.10299550 & $2.8 \mathrm{e}-5$ & 1.69 & 81.38 \\
\hline Ref. Price [14] & 0.10296690 \\
\hline \multicolumn{7}{c}{$S=1, Y=0.8$} \\
\hline$N$ & $M$ & Price & Error & time(s) & cond \\
\hline \hline 128 & 64 & 0.15789424 & $1.1 \mathrm{e}-2$ & 0.006 & 27.03 \\
256 & 128 & 0.15130905 & $3.4 \mathrm{e}-3$ & 0.04 & 56.31 \\
512 & 256 & 0.14852946 & $6.3 \mathrm{e}-4$ & 0.26 & 93.57 \\
1024 & 512 & 0.14789155 & $2.6 \mathrm{e}-6$ & 1.66 & 259.40 \\
\hline Ref. Price $[14]$ & 0.14789424 \\
\hline \multicolumn{7}{c}{}
\end{tabular}

Table 4: Price of European put options with parameter set 2 and truncated domain is $[-2,2]$ and $\epsilon=0.07$ for $Y=0.1$ and $\epsilon=0.11$ for $Y=0.5$ and $Y=0.8$. 


\begin{tabular}{llllllllll}
\hline & $r$ & $\sigma$ & $q$ & $C$ & $G$ & $M$ & $Y$ & $T$ & $K$ \\
\hline CGMY, parameter set 3, see [38] & 0.07 & 0.2 & 0 & 0.5 & 7 & 9 & 1.4 & 0.25 & 10 \\
VG, parameter set 4, see [38] & 0.06 & 0 & 0 & 1 & 8 & 6 & 0 & 1 & 10 \\
CGMY, parameter set 5, see [38] & 0.06 & 0 & 0 & 1 & 10 & 8 & 0.6 & 1 & 10 \\
CGMY, parameter set 6, see [31, 38] & 0.04 & 0.15 & 0 & 0.5 & 10 & 10 & 1.2 & 0.25 & 10 \\
VG, parameter set 7, see [38] & 0.06 & 0 & 0 & 4 & 8 & 8 & 0 & 0.5 & 10 \\
\hline
\end{tabular}

Table 5: Parameter setup for American put options.

We considered parameter set 6 given in Table 5 for American put option and results presented in Table 7. Also, in [31] parameter set 6 has been used for American put option and results reported in Table 5. Comparing results confirm that RBF-PU method needs less time and space steps to reach an specific error level, and definitely it will be faster than the proposed numerical scheme discussed in [31].

To show the convergence of the time discretization, we numerically investigate the behavior of the global temporal errors as a function of $\Delta \tau$ which is defined by

$$
\text { Maximum Error }=\max _{j}\left|u^{m}\left(T, x_{j}\right)-u^{3000}\left(T, x_{j}\right)\right|,
$$

where $u^{m}$ is the numerical solution of European and American options at the spatial nodes $x_{j} \in$ $\left(\log \left(\frac{1}{2}\right), \log \left(\frac{3}{2}\right)\right)$ associated with $S_{j} \in\left(\frac{K}{2}, \frac{3 K}{2}\right)$ after $m$ time steps, and $u^{3000}$ is the corresponding solution for $m=3000$ used as an approximation for the exact solution. Figure 2 displays the global temporal errors versus $\Delta \tau$ for sequence of seven increasing time steps $m$, namely $8,16,32$, 64, 128, 256, 512 and $N=512$ spatial nodes for American and European options. As a first main observation, the global temporal errors decrease monotonically as $M$ increases or equivalently $\Delta \tau$ decreases. Concerning the actual convergence behavior, it is easy to see from Figure 2 that the temporal errors as a function of $\Delta \tau$ are bounded from above in each set of parameter by $C(\Delta \tau)^{p}$ with some moderate constants $C$ where $p \approx 1$ for more cases.

Also, to validate that the RBF-PU method is numerically well-behaved, we present the maximum error convergence with respect to the number of grid points over the region $\left(\frac{K}{2}, \frac{3 K}{2}\right)$ in Figure 3 . The maximum errors presented in 3 show that errors decrease monotonically as $N$ increases or equivalently $\Delta x$ decreases. Concerning the actual convergence behavior, it is easy to see from Figure 3 that the temporal errors as a function of $N$ are bounded from above in each set of parameter by $C(N)^{-p}$ with some moderate constants $C$ where $p \approx 2$ for more cases.

\subsection{Binary barrier and butterfly options}

One of the advantages of the RBF-PU method as presented in this study is that one can deal with complicated payoffs such as binary barrier and butterfly options without affecting the accuracy. The numerical experiments confirm this fact. Therefore, in this subsection, we consider a European binary down-and-out barrier option that is used to pricing a credit default swap by Cariboni and Schoutens in [11]. The payoff function of the binary down-and-out barrier option is given by

$$
g(x)=\mathbf{1}_{\left\{x>\ln \left(\frac{B}{K}\right)\right\}},
$$

where the value $B$ is a barrier level and $\mathbf{1}$ is the indicator function. 


\begin{tabular}{cccccc}
\hline \multicolumn{7}{c}{$S=10$, parameter set 3 } \\
\hline$N$ & $M$ & Price & Error & time(s) & cond \\
\hline 128 & 64 & 1.216663 & $5.2 \mathrm{e}-2$ & 0.006 & 10.11 \\
256 & 128 & 1.251458 & $1.8 \mathrm{e}-2$ & 0.04 & 24.46 \\
512 & 256 & 1.268218 & $1.2 \mathrm{e}-3$ & 0.26 & 50.71 \\
1024 & 512 & 1.269537 & $2.1 \mathrm{e}-5$ & 1.64 & 99.56 \\
\hline Ref. Price [32] & 1.269516 \\
\hline \hline \multicolumn{5}{c}{$S=10$, parameter set 4 } \\
\hline$N$ & $M$ & Price & Error & time(s) & cond \\
\hline 128 & 64 & 0.489189 & $6.6 \mathrm{e}-3$ & 0.006 & 2.33 \\
256 & 128 & 0.497642 & $1.7 \mathrm{e}-3$ & 0.03 & 2.71 \\
512 & 256 & 0.496501 & $6.2 \mathrm{e}-4$ & 0.26 & 3.70 \\
1024 & 512 & 0.495835 & $3.9 \mathrm{e}-5$ & 1.67 & 6.91 \\
\hline Ref. Price [32] & 0.495875 & & \\
\hline \hline \multicolumn{7}{c}{$S=10$, parameter set 5} \\
\hline$N$ & $M$ & Price & Error & time(s) & cond \\
\hline 128 & 64 & 0.812406 & $6.9 \mathrm{e}-2$ & 0.006 & 4.11 \\
256 & 128 & 0.875805 & $6.1 \mathrm{e}-3$ & 0.04 & 9.07 \\
512 & 256 & 0.881678 & $2.7 \mathrm{e}-4$ & 0.25 & 19.05 \\
1024 & 512 & 0.881959 & $1.0 \mathrm{e}-5$ & 1.63 & 38.57 \\
\hline Ref. Price $[32]$ & 0.881949 & & \\
\hline \multicolumn{7}{c}{}
\end{tabular}

Table 6: Price of American put options with parameter sets 3,4 and 5 with truncated domain is $[-3,3]$ and $\epsilon=0.09$.
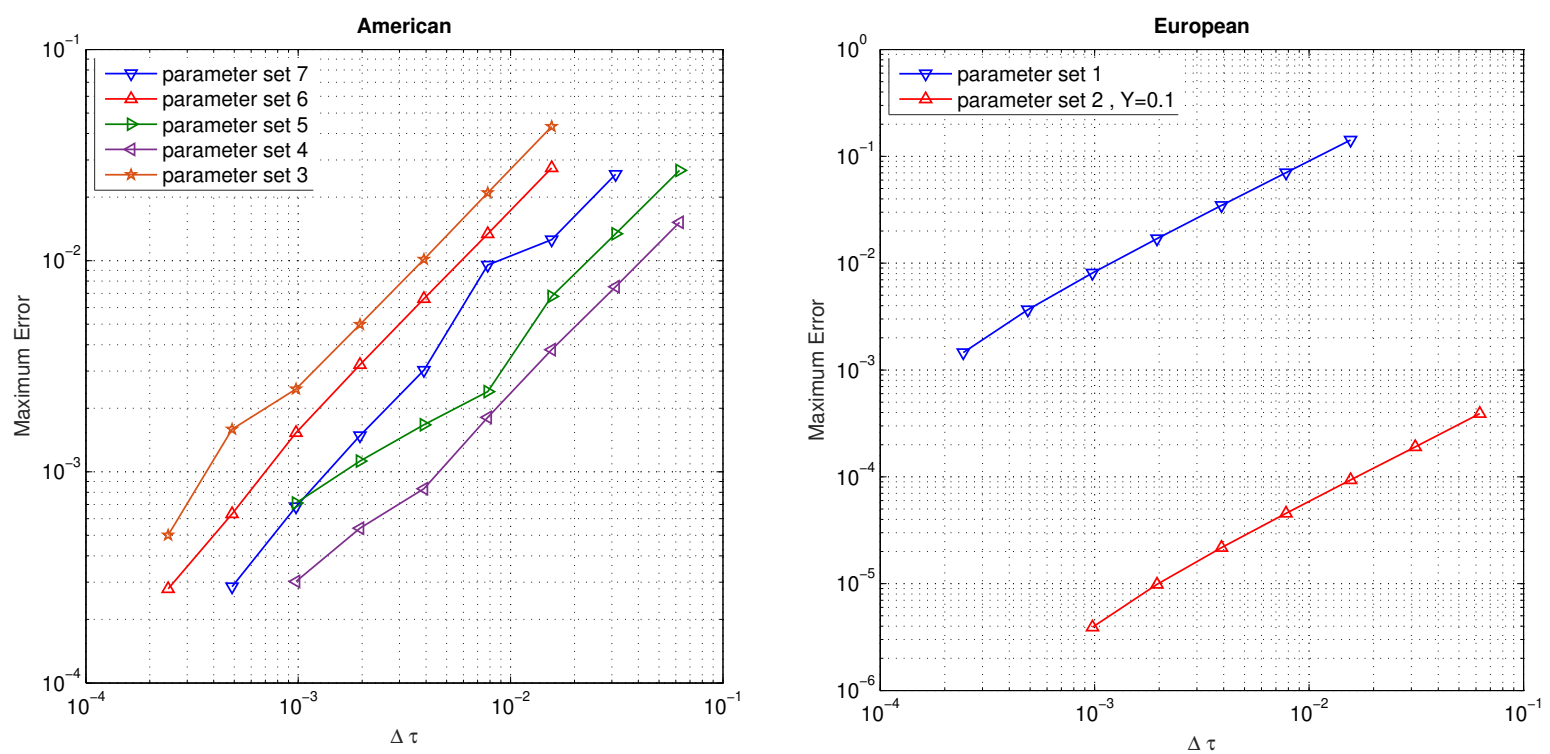

Figure 2: Temporal error vs. $\Delta \tau$ with $N=512$ spatial nodes for American and European options. 


\begin{tabular}{|c|c|c|c|c|c|c|c|c|c|}
\hline \multicolumn{10}{|c|}{ parameter sets 6} \\
\hline & & \multicolumn{2}{|c|}{$S=8$} & \multicolumn{2}{|c|}{$S=10$} & \multicolumn{2}{|c|}{$S=12$} & & \\
\hline$N$ & $M$ & Price & Error & Price & Error & Price & Error & time $(\mathrm{s})$ & cond \\
\hline 128 & 64 & 2.105294 & $1.1 \mathrm{e}-2$ & 0.841094 & $8.9 \mathrm{e}-3$ & 0.268801 & $2.3 \mathrm{e}-3$ & 0.08 & 4.80 \\
\hline 256 & 128 & 2.093481 & $2.3 \mathrm{e}-2$ & 0.843376 & $6.6 \mathrm{e}-3$ & 0.267985 & $3.1 \mathrm{e}-3$ & 0.13 & 10.79 \\
\hline 512 & 256 & 2.115072 & $1.7 \mathrm{e}-3$ & 0.849632 & $3.6 \mathrm{e}-4$ & 0.270562 & $6.0 \mathrm{e}-4$ & 0.25 & 22.82 \\
\hline 1024 & 512 & 2.116874 & $9.7 \mathrm{e}-5$ & 0.850082 & $8.6 e-5$ & 0.271122 & $4.2 \mathrm{e}-5$ & 1.69 & 45.70 \\
\hline \multicolumn{2}{|c|}{ Ref. Price [32] } & 2.116776 & & 0.849996 & & 0.271165 & & & \\
\hline \multicolumn{10}{|c|}{ parameter sets 7} \\
\hline & & \multicolumn{2}{|c|}{$S=9$} & \multicolumn{2}{|c|}{$S=10$} & \multicolumn{2}{|c|}{$S=11$} & & \\
\hline$N$ & $M$ & Price & Error & Price & Error & Price & Error & time $(\mathrm{s})$ & cond \\
\hline 128 & 64 & 1.292267 & $3.4 \mathrm{e}-2$ & 0.780565 & $2.0 \mathrm{e}-2$ & 0.458218 & $1.3 \mathrm{e}-2$ & 0.003 & 2.54 \\
\hline 256 & 128 & 1.322932 & $3.4 \mathrm{e}-3$ & 0.798194 & $2.4 \mathrm{e}-3$ & 0.468734 & $2.4 \mathrm{e}-3$ & 0.03 & 3.38 \\
\hline 512 & 256 & 1.325914 & $4.7 \mathrm{e}-4$ & 0.800872 & $2.7 \mathrm{e}-4$ & 0.470656 & $5.6 \mathrm{e}-4$ & 0.24 & 6.40 \\
\hline 1024 & 512 & 1.326338 & $4.6 \mathrm{e}-5$ & 0.801404 & $8.0 \mathrm{e}-4$ & 0.471168 & $5.3 \mathrm{e}-5$ & 1.68 & 12.88 \\
\hline Ref. & ce [32] & 1.326384 & & 0.800601 & & 0.471222 & & & \\
\hline
\end{tabular}

Table 7: Price of American put options with parameter sets 6 and 7 with truncated domain is $[-3,3]$ and $\epsilon=0.09$.

The corresponding parameters under the variance gamma model are given by

$$
\begin{gathered}
r=0.0421, \quad \sigma=0, \quad q=0, \quad C=\frac{1}{\hat{v}}, \quad B=50, \quad T=1, \quad K=100 \\
G=\left(\sqrt{\frac{\hat{\theta}^{2} \hat{v}^{2}}{4}+\frac{\hat{\sigma}^{2} \hat{v}}{2}}-\frac{\hat{\theta} \hat{v}}{2}\right)^{-1}, \quad M=\left(\sqrt{\frac{\hat{\theta}^{2} \hat{v}^{2}}{4}+\frac{\hat{\sigma}^{2} \hat{v}}{2}}+\frac{\hat{\theta} \hat{v}}{2}\right)^{-1}, \quad Y=0
\end{gathered}
$$

where $\hat{\sigma}=0.20722, \hat{v}=0.50215$, and $\hat{\theta}=-0.22898$. These parameters are also taken in [11]. We use the truncated domain $[-2,2]$ of the log price.

In [11] authors used the numerical method developed in [27] for pricing a binary down-andin barrier option under the variance gamma model. The price of the binary down-and-in barrier option in [11] is 0.0253 at $S=100$. Then the reference value of the binary down-and-out barrier option is 0.933474 at $S=100$ according to the relationship between two options [31].

Numerical results for European binary down-and-out barrier option obtained by RBF-PU method are given in Table 8 including option price and absolute error at $S=K=100$, CPU times in second and condition number of arisen linear system of equations. These parameters have been used in [31] for evaluating values of European binary down-and-out barrier option by the implicit method with three time levels and results presented in Table 6. Comparing results clearly confirm that for specific error level, the proposed RBF-PU method needs less number of time and spatial steps. Also, the option value for European binary down-and-out barrier option is plotted in Figure 4.

Now, we consider European and American butterfly options to check the efficiency and accuracy of developed meshfree method. The reason for including a butterfly option is to show that the 

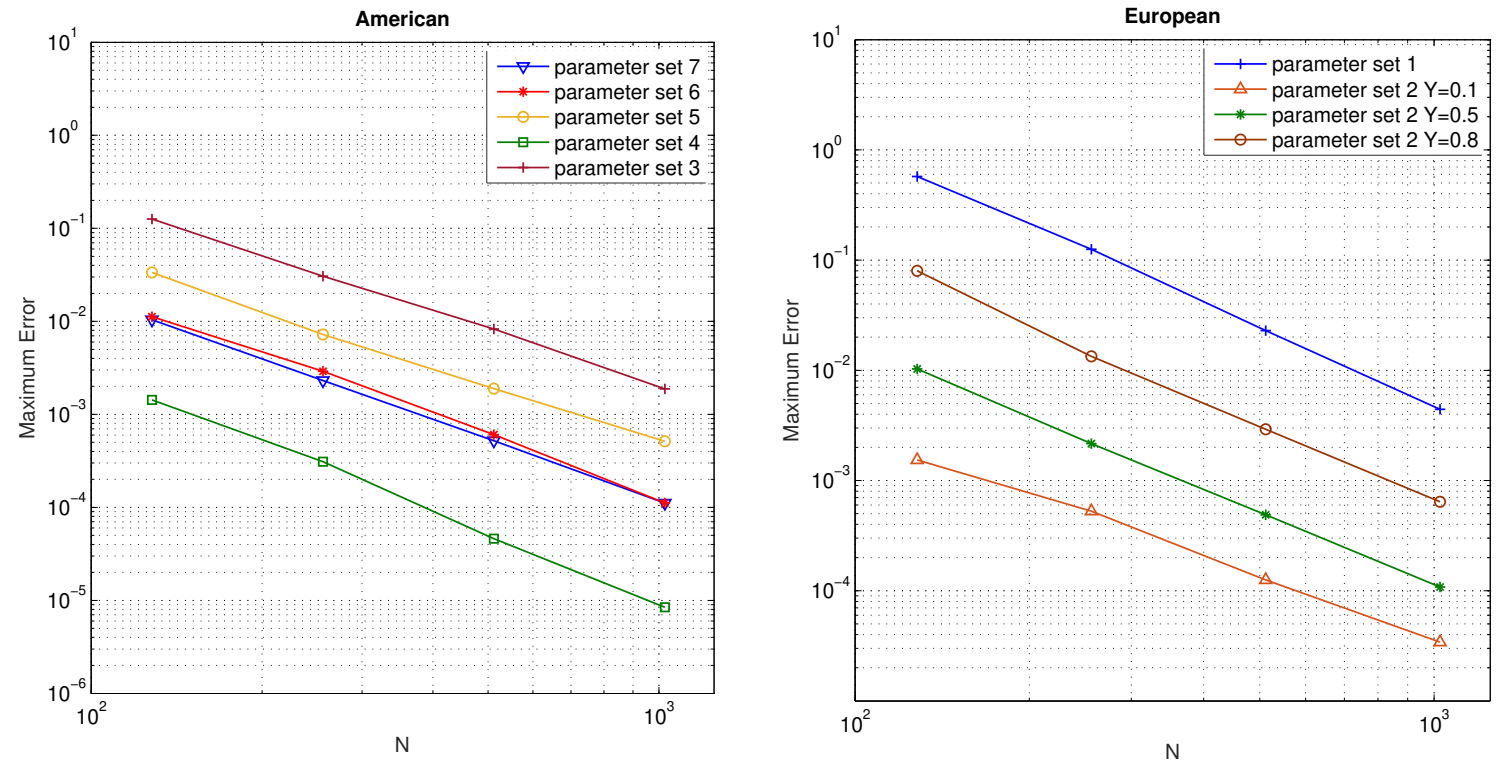

Figure 3: Maximum error convergence with respect to the number of points for American and European options. The Maximum error is measured over the region $\left(\frac{K}{2}, \frac{3 K}{2}\right)$ with $N=3000$ and $M=512$ as reference solution.

\begin{tabular}{cccccc}
\hline \multicolumn{6}{c}{$S=100$} \\
\hline$N$ & $M$ & Price & Error & time(s) & cond \\
\hline 128 & 64 & 0.945296 & $1.1 \mathrm{e}-2$ & 0.006 & 7.12 \\
256 & 128 & 0.943196 & $9.7 \mathrm{e}-3$ & 0.04 & 15.30 \\
512 & 256 & 0.943335 & $9.8 \mathrm{e}-3$ & 0.25 & 25.51 \\
1024 & 512 & 0.933494 & $2.4 \mathrm{e}-5$ & 1.63 & 50.43 \\
\hline \multicolumn{7}{l}{ Ref. Price [31] } & 0.933474 & & & \\
\hline
\end{tabular}

Table 8: Price of European binary down-and-out barrier option with truncated domain is $[-2,2]$ and $\epsilon=0.07$. 

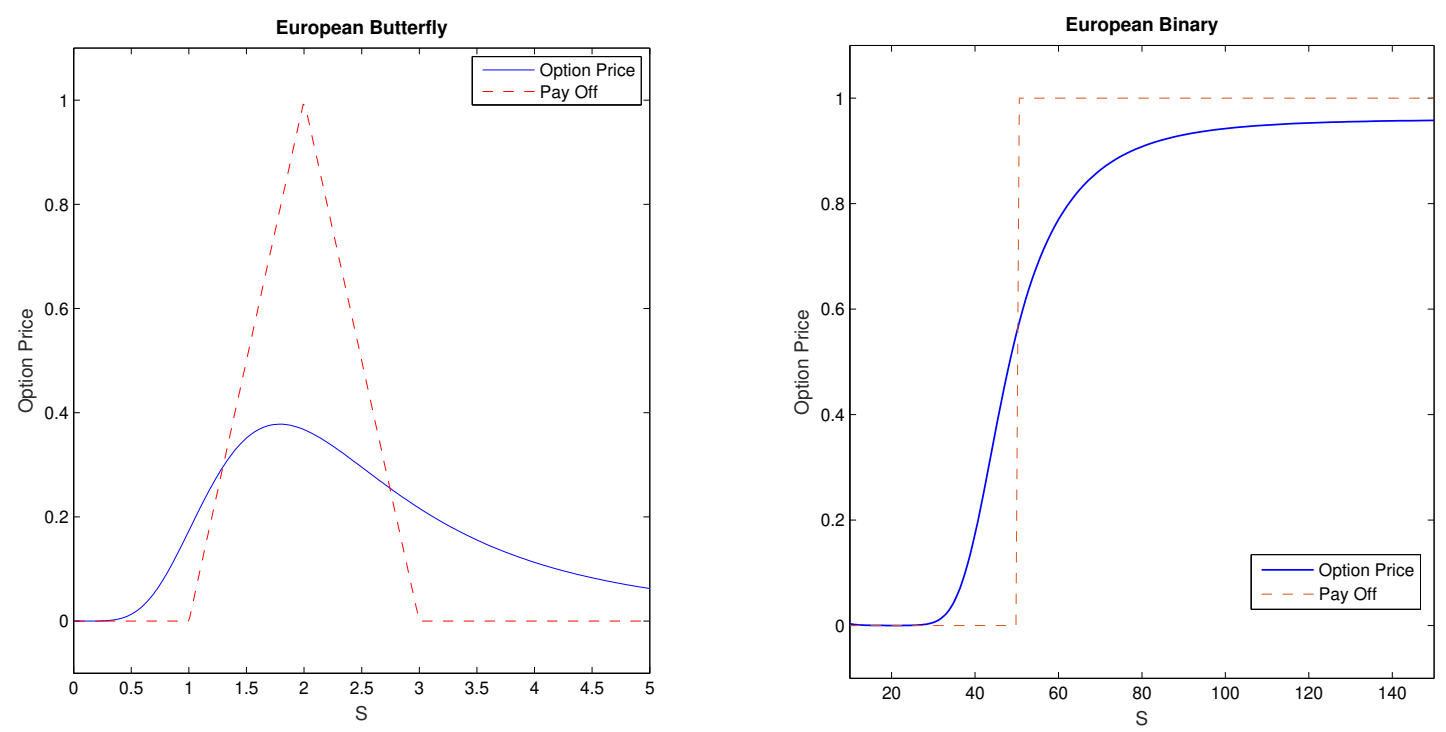

Figure 4: Left: The option value for European butterfly option. Right: The option value for European binary down-and-out barrier option.

method discussed in this paper is rather applicable and provides accurate numerical results for non-convex payoffs. A butterfly spread is the result of buying call options with strikes $K_{1}$ and $K_{2}$ respectively, and selling two call options with strike price $K_{3}=\frac{K_{1}+K_{2}}{2}$, Then, the payoff function will be

$$
g(x)=\max \left(e^{x}-K_{1}, 0\right)-2 \max \left(e^{x}-K_{3}, 0\right)+\max \left(e^{x}-K_{2}, 0\right) .
$$

A European style butterfly is appropriate when the investor thinks large moves in the price of the underlying asset are unlikely. It usually has a middle strike $K_{3}$ around the spot price $K$ of the underlying asset and it pays off when the price of the underlying asset stays close to $K$. Numerical results for European and American butterfly options with parameters:

$$
r=0.1, \quad \sigma=0, \quad q=0, \quad K=1, \quad T=1, \quad C=1, \quad G=3, \quad M=5, \quad Y=0.5
$$

obtained by RBF-PU method are given in Table 9 including option price and absolute error at $S=K=1$, CPU times in second and condition number of arisen linear system of equations. Also, in Table 10, we report $l_{\infty}$-errors to show the accuracy of RBF-PU method in the region of interest $\left[\frac{K}{2}, \frac{3 K}{2}\right]$ not only on the specific point such as $S=K$, and for evaluating these errors we use Carr-Madan formula [14]. The option value for European butterfly is plotted in Figure 4.

\subsection{Greeks}

In this section our aim is to evaluate the efficiency of the RBF-PU method for computing the Greeks $\Delta=\frac{\partial v}{\partial S}$ and $\Gamma=\frac{\partial^{2} v}{\partial S^{2}}$ which are important for hedging the option. Delta measures the sensitivity of the option price to a fluctuation in the underlying asset price while Gamma is the speed at which the Delta changes with respect to this movement. We plotted in Figures 6 and 7 the Delta and 


\begin{tabular}{cccccccccc}
\hline \multicolumn{9}{c}{ European } & \multicolumn{5}{c}{ American } \\
\hline$N$ & $M$ & Price & Error & time(s) & cond & Price & Error & time(s) & cond \\
\hline 128 & 64 & 0.1766315 & $3.5 \mathrm{e}-3$ & 0.003 & 13.98 & 0.1994502 & $6.6 \mathrm{e}-3$ & 0.006 & 3.84 \\
256 & 128 & 0.1738725 & $8.2 \mathrm{e}-4$ & 0.04 & 29.87 & 0.2048898 & $1.1 \mathrm{e}-3$ & 0.04 & 8.48 \\
512 & 256 & 0.1732450 & $2.0 \mathrm{e}-4$ & 0.27 & 58.54 & 0.2059969 & $5.9 \mathrm{e}-5$ & 0.24 & 18.02 \\
1024 & 512 & 0.1730752 & $3.0 \mathrm{e}-5$ & 1.64 & 147.69 & 0.2060638 & $7.0 \mathrm{e}-6$ & 1.70 & 36.66 \\
\hline \multicolumn{2}{l}{ Ref. Price $[14]$} & 0.1730448 & & & & 0.2060568 & & & \\
\hline
\end{tabular}

Table 9: Price of European and American butterfly options at $S=1$ with truncated domain $[-3,3]$ and $\epsilon=0.15$ for European option and $\epsilon=0.09$ for American option.

\begin{tabular}{cccc}
\hline & \multicolumn{2}{c}{$l_{\infty}$-errors } \\
\hline$N$ & $M$ & European & American \\
\hline 128 & 128 & $1.6 \mathrm{e}-2$ & $2.2 \mathrm{e}-2$ \\
256 & 256 & $5.1 \mathrm{e}-3$ & $1.1 \mathrm{e}-2$ \\
512 & 512 & $1.5 \mathrm{e}-3$ & $6.2 \mathrm{e}-3$ \\
1024 & 1024 & $6.8 \mathrm{e}-4$ & $1.9 \mathrm{e}-3$ \\
\hline
\end{tabular}

Table 10: $l_{\infty}$-errors for European and American butterfly options with truncated domain $\left[\frac{K}{2}, \frac{3 K}{2}\right]$ and $\epsilon=0.15$ for European option and $\epsilon=0.09$ for American option.

Gamma functions against the reference solutions for European and American butterfly options. In these figures reference solutions are derived from Carr-Madan formula [14].

\subsection{Properties of the RBF-PU discretization matrices}

If we use global RBF approximation coefficient matrix is dense ordinary and condition number is very large. Using partition of unity method of RBF introduces sparsity in the discretization matrices. In Figure 8 the sparsity pattern of the discretized matrix in RBF-PU is shown. Also, we considered global RBF method for discretization of (4) and condition number of discretized matrix is given in Table 11 for different sets of parameter and space step sizes. Comparing results with condition number of localized RBF-PU method shows that linear system of equations arisen in RBF-PU is well conditioned.

\section{Conclusion}

We proposed RBF-PU method for spatial discretization of PIDE to price American and European options under Lévy model. Also, penalty method is used to solve the free boundary problem arisen in pricing American option. Then, CNLF is applied for time discretisations and we treat the local term using an implicit step and the nonlocal term using an explicit step to avoid to avoid the inversion of the nonsparse matrix. These result in a linear algebraic system with a sparse matrix that has a small condition number. The shape parameter in the RBF affects the accuracy and stability of the numerical methods. Figure 1 confirms that RBF-PU method is less sensitive to the change of shape parameter. An increase of the number of nodal points and correspondingly an increase 

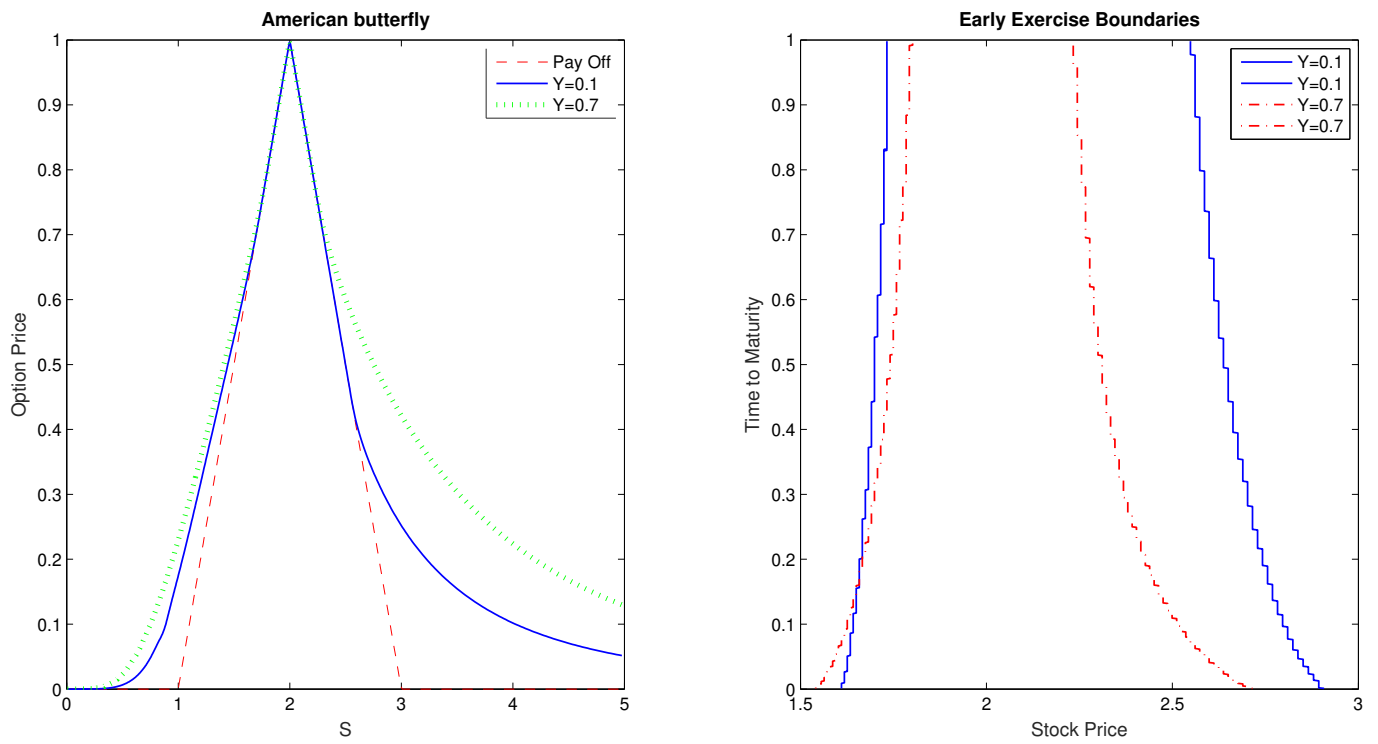

Figure 5: Left: The option value for American butterfly option. Right: Early exercise boundaries for American butterfly option with $Y=0.1$ and $Y=0.7$.
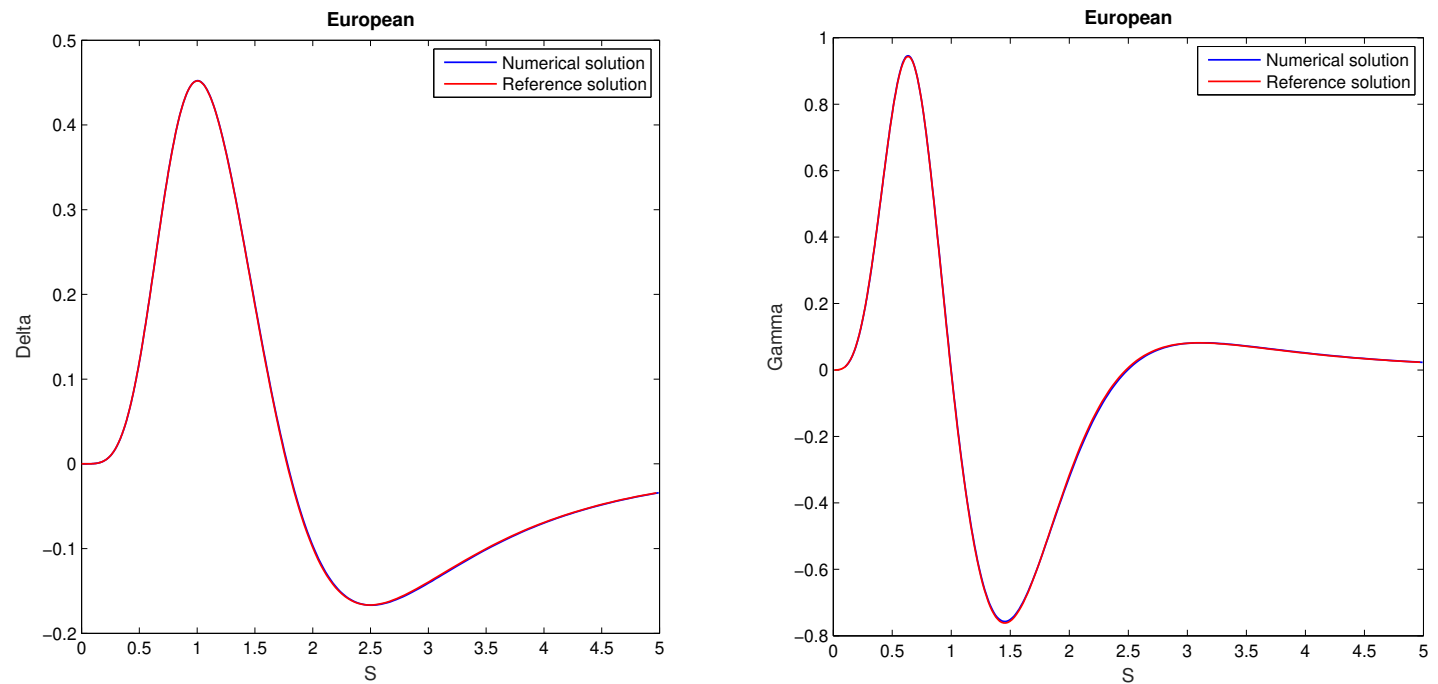

Figure 6: Delta and Gamma of European butterfly option against the reference solutions. 

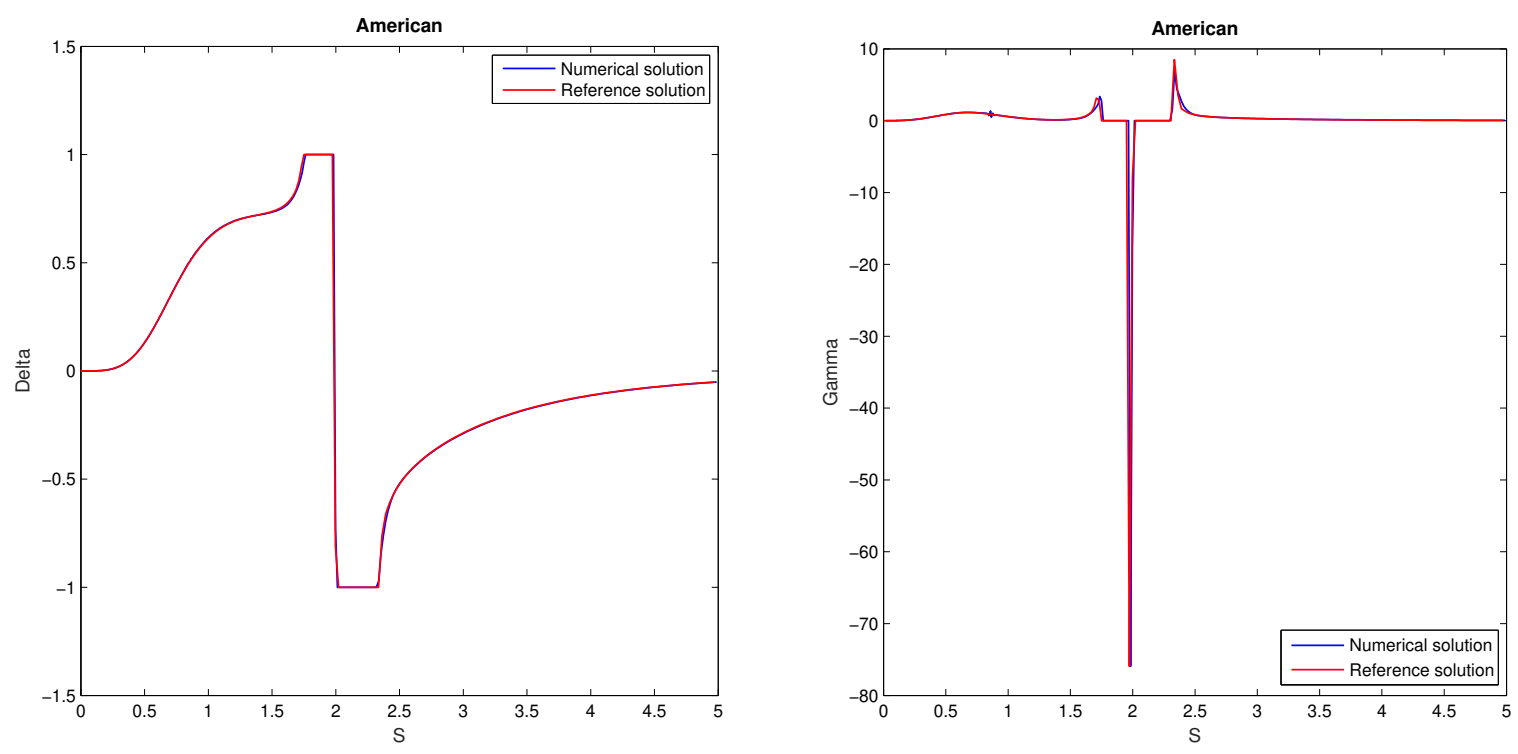

Figure 7: Delta and Gamma of American butterfly option against the reference solutions.

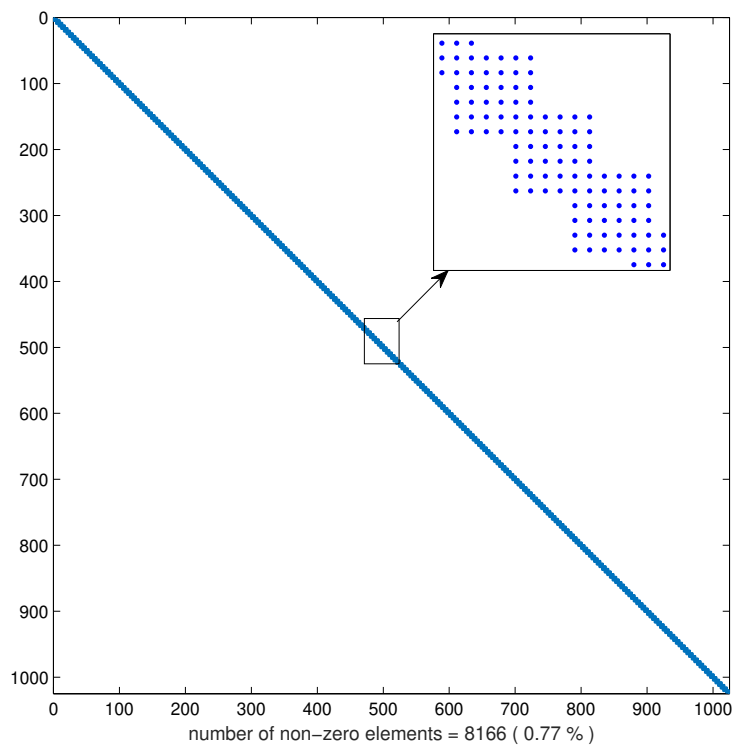

Figure 8: The uniform grid with 1024 computational nodes. The partition of unity is performed using 256 circular patches of equal size. The RBF-PU coefficient matrix that was obtained on this grid. It contains only 8166 non-zero elements. 


\begin{tabular}{lcc}
\hline & Condition number of RBF-PU & Condition number of global RBF \\
\hline parameter set 1 & 617.86 & $2.97 \mathrm{e} 20$ \\
parameter set 2, $Y=0.1$ & 12.57 & $1.31 \mathrm{e} 20$ \\
parameter set 2, $Y=0.5$ & 81.38 & $1.27 \mathrm{e} 20$ \\
parameter set 2, $Y=0.8$ & 259.40 & $7.26 \mathrm{e} 20$ \\
parameter set 3 & 99.56 & $1.44 \mathrm{e} 21$ \\
parameter set 4 & 6.91 & $1.15 \mathrm{e} 20$ \\
parameter set 5 & 38.57 & $6.46 \mathrm{e} 20$ \\
parameter set 6 & 45.70 & $2.34 \mathrm{e} 20$ \\
parameter set 7 & 12.88 & $1.49 \mathrm{e} 21$ \\
\hline
\end{tabular}

Table 11: Condition number of localized RBF-PU method and global RBF method for different sets of parameter and $N=1024$ as space step size.

of the number of patches in RBF-PU method also leads to an improvement of the approximation. The effect of the time discretization is measured by studying the temporal error. For the American and European option cases where the CNLF scheme is combined with penalty method, we conclude that the rate of convergence is of at least first order in time for more cases. Also, Figure 3 confirms that our scheme is stable and second order convergent in space. The experiments also demonstrated that European and American option prices with error around 1.0e-4 can be computed in less than one second on a PC. Thus, the developed method is very fast and accurate.

\section{Acknowledgements}

The authors would like to thank professor Ariel Almendral Vazquez, Senior Research Scientist SAND, Norwegian Computing Center, for the provided reference solution for European and American butterfly options and some fruitful comments. 


\section{References}

[1] A. Almendral and C. Oosterlee. Accurate evaluation of European and American options under the CGMY process. SIAM Journal on Scientific Computing, 29(1):93-117, 2007.

[2] Leif Andersen and Jesper Andreasen. Jump-diffusion processes: Volatility smile fitting and numerical methods for option pricing. Review of Derivatives Research, 4(3):231-262, 2000.

[3] Søren Asmussen and Jan Rosiński. Approximations of small jumps of Lévy processes with a view towards simulation. Journal of Applied Probability, 38(2):482-493, 2001.

[4] I. Babuška and J.M. Melenk. The partition of unity method. International Journal for Numerical Methods in Engineering, 40(4):727 - 758, 1997.

[5] D.S. Bates. Jumps and stochastic volatility: Exchange rate processes implicit in Deutsche mark options. The Review of Financial Studies, 9(1):69-107, 1996.

[6] F. Black and M. Scholes. The pricing of options and corporate liabilities. Journal of Political Economy, 81(3):637 - 654, 1973.

[7] A. Borici and H.-J. Lüthi. Pricing American put options by fast solutions of the linear complementarity problem. In E.J. Kontoghiorghes, B. Rustem, and S. Siokos, editors, Computational Methods in Decision-Making, Economics and Finance, pages 325 - 338. Springer US, 2002.

[8] Maya Briani, Roberto Natalini, and Giovanni Russo. Implicit-explicit numerical schemes for jump-diffusion processes. Calcolo, 44(1):33-57, 2007.

[9] M.D. Buhmann. Radial basis functions. Acta Numerica, 9:1 - 38, 012000.

[10] M.D. Buhmann. Radial basis functions: theory and implementations. Cambridge University Press, New York, 2003.

[11] Jessica Cariboni and Wim Schoutens. Pricing credit default swaps under Lévy models. Journal of Computational Finance, 10:71-91, 2007.

[12] P. Carr and H. Geman. The fine structure of asset returns: An empirical investigation. The Journal of Business, 75(2):305-332, 2002.

[13] Peter Carr, Hélyette Geman, Dilip B Madan, and Marc Yor. The fine structure of asset returns: An empirical investigation. The Journal of Business, 75(2):305-332, 2002.

[14] Peter Carr and Dilip B. Madan. Option valuation using the fast fourier transform. Journal of Computational Finance, 2:61-73, 1999.

[15] R. Cavoretto and A. De Rossi. Spherical interpolation using the partition of unity method: An efficient and flexible algorithm. Applied Mathematics Letters, 25(10):1251 - 1256, 2012.

[16] R. Cavoretto and A. De Rossi. A meshless interpolation algorithm using a cell-based searching procedure. Computers $\&$ Mathematics with Applications, 67(5):1024 - 1038, 2014.

[17] Simon S. Clift and Peter A. Forsyth. Numerical solution of two asset jump diffusion models for option valuation. Applied Numerical Mathematics, 58(6):743 - 782, 2008. 
[18] R. Cont and P. Tankov. Financial Modelling with Jump Processes. Chapman \& Hall, Cambridge, 2004.

[19] Rama Cont and Ekaterina Voltchkova. A finite difference scheme for option pricing in jump diffusion and exponential Lévy models. SIAM Journal on Numerical Analysis, 43(4):15961626, 2005.

[20] Y. d'Halluin, P. A. Forsyth, and K. R. Vetzal. Robust numerical methods for contingent claims under jump diffusion processes. IMA Journal of Numerical Analysis, 25(1):87, 2005.

[21] Y. d'Halluin, P.A. Forsyth, and G. Labahn. A penalty method for American options with jump diffusion processes. Numerische Mathematik, 97(2):321-352, 2004.

[22] Bjrn Eraker, Michael Johannes, and Nicholas Polson. The impact of jumps in volatility and returns. The Journal of Finance, 58(3):1269-1300, 2003.

[23] F. Fang and C. W. Oosterlee. A novel pricing method for European options based on fouriercosine series expansions. SIAM Journal on Scientific Computing, 31(2):826-848, 2009.

[24] F. Fang and C. W. Oosterlee. Pricing early-exercise and discrete barrier options by fouriercosine series expansions. Numerische Mathematik, 114(1):27, Aug 2009.

[25] G.E. Fasshauer. Meshfree Approximation Methods with Matlab. World Scientific Publishing Co, Singapore, 2007.

[26] G.E. Fasshauer, A.Q.M. Khaliq, and D.A. Voss. Using meshfree approximation for multiasset American options. Journal of the Chinese Institute of Engineers, 27(4):563 - 571, 2004.

[27] Ali Hirsa and Dilip B. Madan. Pricing American options under variance gamma. Journal of Computational Finance, 7(2):63-80, 2003.

[28] Yiu-Chung Hon and Xian zhong Mao. A radial basis function method for solving options pricing model. Financial Engineering, 8:31-49, 1999.

[29] S. Ikonen and J. Toivanen. Operator splitting methods for American option pricing. Applied Mathematics Letters, 17(7):809 - 814, 2004.

[30] S.G. Kou. A jump-diffusion model for option pricing. Management Science, 48(8):1086-1101, 2002 .

[31] Jaewook Lee and Younhee Lee. Tridiagonal implicit method to evaluate European and American options under infinite activity Lévy models. Journal of Computational and Applied Mathematics, 237(1):234 - 243, 2013.

[32] R. Lord, F. Fang, F. Bervoets, and C. W. Oosterlee. A fast and accurate FFT-based method for pricing early-exercise options under Lévy processes. SIAM Journal on Scientific Computing, 30(4):1678-1705, 2008.

[33] Dilip B. Madan and Eugene Seneta. The variance gamma (V.G.) model for share market returns. The Journal of Business, 63(4):511-524, 1990. 
[34] A.-M. Matache, P.-A. Nitsche, and C. Schwab. Wavelet galerkin pricing of American options on Lévy driven assets. Quantitative Finance, 5(4):403-424, 2005.

[35] R. Merton. Option pricing when underlying stock returns are discontinuous. Journal of Financial Economics, 3(1-2):125-144, 1976.

[36] Reza Mollapourasl, Ali Fereshtian, Hengguang Li, and Xun Lu. RBF-PU method for pricing options under the jump-diffusion model with local volatility. Journal of Computational and Applied Mathematics, 337:98 - 118, 2018.

[37] Reza Mollapourasl, Ali Fereshtian, and Michèle Vanmaele. Radial basis functions with partition of unity method for American options with stochastic volatility. Computational Economics, Sep 2017.

[38] Rambeerich Nisha, Tangman Desire Yannick, and Bhuruth Muddun. Numerical pricing of American options under infinite activity Lévy processes. Journal of Futures Markets, 31(9):809-829, 2011.

[39] U. Pettersson, E. Larsson, G. Marcusson, and J. Persson. Improved radial basis function methods for multi-dimensional option pricing. Journal of Computational and Applied Mathematics, 222(1):82 - 93, 2008. Special Issue: Numerical \{PDE\} Methods in Finance.

[40] M.J.D. Powell. The theory of radial basis functions approximation in 1990. In W.A. Light, editor, Advances in Numerical Analysis. Vol. II. Wavelets, Subdivision Algorithms and Radial Basis Functions, page 105 210. Oxford Univ. Press, London, 1992.

[41] A. Safdari-Vaighani, A. Heryudono, and E. Larsson. A radial basis function partition of unity collocation method for convection-diffusion equations arising in financial applications. Journal of Scientific Computing, 64(2):341 - 367, 2015.

[42] Robert Schaback. A unified theory of radial basis functions. Journal of Computational and Applied Mathematics, 121(1):165 - 177, 2000.

[43] R. Seydel. Tools for Computational Finance. Springer, Berlin, Heidelberg, 4th edition, 2009.

[44] V. Shcherbakov and E. Larsson. Radial basis function partition of unity methods for pricing vanilla basket options. Computers $\&$ Mathematics with Applications, 71(1):185 - 200, 2016.

[45] D. Shepard. A two-dimensional interpolation function for irregularly-spaced data. In Proceedings of the 1968 23rd ACM National Conference, ACM '68, pages 517 - 524, New York, NY, USA, 1968. ACM.

[46] Jari Toivanen. Numerical valuation of european and american options under kou's jumpdiffusion model. SIAM Journal on Scientific Computing, 30(4):1949-1970, 2008.

[47] Iris R. Wang, Justin W. L. Wan, and Peter A. Forsyth. Robust numerical valuation of European and American options under the CGMY process. Journal of Computational Finance, 10(4):3169, 2007.

[48] H. Wendland. Piecewise polynomial, positive definite and compactly supported radial functions of minimal degree. Advances in Computational Mathematics, 4(1):389 - 396, 1995. 
[49] H. Wendland. Fast evaluation of radial basis functions: Methods based on partition of unity. In C.K. Chui, L.L. Schumaker, and J. Stöckler, editors, Approximation Theory X: Wavelets, Splines, and Applications, pages 473 - 483. Vanderbilt University Press, Nashville, 2002.

[50] H. Wendland. Scattered Data Approximation. Number 17 in Cambridge Monographs on Applied and Computational Mathematics. Cambridge University Press, New York, 2005.

[51] Z. Wu and Y.C. Hon. Convergence error estimate in solving free boundary diffusion problem by radial basis functions method. Engineering Analysis with Boundary Elements, 27(1):73 79, 2003.

[52] R. Zvan, P.A. Forsyth, and K.R. Vetzal. Penalty methods for American options with stochastic volatility. Journal of Computational and Applied Mathematics, 91(2):199 - 218, 1998. 